\title{
The Impact of Bullwhip Effect on the Cash Flow in Two-Parallel Supply Chain Systems with the Competition Effect
}

\author{
Xingji Chen, ${ }^{1}$ Jing Zeng, ${ }^{1}$ and Xigang Yuan ${ }^{2}{ }^{2}$ \\ ${ }^{1}$ Luzhou Vocational and Technical College, Luzhou 646000, China \\ ${ }^{2}$ Business School, Jiangsu Normal University, Xuzhou 221116, China \\ Correspondence should be addressed to Xigang Yuan; yxg200811606@126.com
}

Received 29 July 2021; Revised 28 October 2021; Accepted 15 December 2021; Published 15 January 2022

Academic Editor: Sundarapandian Vaidyanathan

Copyright (c) 2022 Xingji Chen et al. This is an open access article distributed under the Creative Commons Attribution License, which permits unrestricted use, distribution, and reproduction in any medium, provided the original work is properly cited.

While considering the competition effect and market share, this study discusses how the cash flow bullwhip effect (CFBE) is impacted in two-product and two-parallel supply chain systems by comparing the situation that it has one kind of product in twolevel supply chain (SC). Specifically, the study aimed to examine two-product and two-parallel SC systems that include two suppliers and two retailers. Assuming that the demand function is a linear relationship of price self-sensitivity coefficient and price cross-sensitivity coefficient, which is an $\mathrm{AR}(1)$ process, two retailers share the demand. After that, the quantitative equation of the CFBE was deduced from two-product and two-parallel SC systems. Finally, we get the condition that the competition effect and the market share increase or decrease the CFBE, which was in contrast to the situation without the competition effect and the market share. The paper suggested that the manager can cooperate with their partner if two products are substitutable. On the other hand, the firm should improve the forecasting accuracy of the customer's demand and improve the service quality so that it can increase the market share and reduce the CFBE in two-parallel SC systems.

\section{Introduction}

The amplification of demand information is the most significant barrier getting harmony and keeping development in various levels in SC and which is called as bullwhip effect (BE). It is widely used to study the BE in simple two-stage SC structural model (e.g., Lee et al. [1]; Chen et al. [2]; Chen et al. [3]; Luong [4], and Luong and Phien [5]). While the two-stage SC model has been traditionally using address the issue of the $\mathrm{BE}$, the economic and societal developments that have occurred, which have affected many enterprises (e.g., electronic firms, mobile phones, and so on), it is essential for us to develop a new equation that reflects the chain-to-chain competition effect that the enterprise is not only impacted by others that belong to the same SC but also influenced by enterprises that belong to the other supply chain. This phenomenon can influence upon the two-level SC's efficiency. The competition effect means that it is difficult to determine whether the firms will benefit or not from the BE. Based on the assumption of the two-stage SC model, several studies (e.g., Zhang et al. [6]; Shi et al. [7]; Chen et al. [2]; Chen et al. [3]; Zhang [8]; and Liu [9]) have discussed the coordination and information sharing issue in SC. However, none of the aforementioned studies explored any ideas concerning the BE in two-parallel SC systems. This paper discusses the effect of the competition effect, and market share on the CFBE in two-parallel SC systems.

In this study, the CFBE was used as a method of the SC performance, and this study considered industries with twoparallel SC systems that showed the competition effect in reference to the demand level. Many enterprises met the standard of the model. In the mobile phone firm, this included companies such as Huawei and Apple, and each of them could develop a chain relation with down retailer sharing the same customer's demand. We are aware that the mobile phone's retailing price is a very important factor that impacts demand. The higher the Huawei mobile phone's retailing price is, the lower its market demand is, and vice versa. The product substitutability of Huawei and Apple, if the Apple mobile phone's retailing price is so high, some consumers can turn to Huawei, and its market share increases and vice versa. 
This paper aimed to discuss how the CFBE was impacted in two-product and two-parallel SC systems, which could be contrasted with a single product in the simple two-level SC. For this purpose, we constructed two-parallel SC systems, each of which sells one kind of product including one supplier and one retailer. The demand is the linear function for price self-sensitivity coefficient and the price crosssensitivity coefficient. The price followed AR(1) process and assumed that two retailers share the market. Similarly, because the two products were substitutable, the market demand in each SC depended not only on its own price but also on the price in other SC. Assuming that two retailers ordered products from the supplier, and forecast the consumer's market demand using the moving average forecasting technique (MA). We discuss the influence of their competition effect and market share on the CFBE, while furthermore exploring the condition that the CFBE was enlarged (or reduced).

The remainder of this paper is organized as follows: Section 2 reviews the relevant literature. Section 3 presents the demand process, the order policy, and the moving average forecasting method for each supply chain. Section 4 deduces the bullwhip effect (BE), the IBE, and the CFBE. Section 5 discusses the effect of the relevant factors on CFBE. The numerical analysis is introduced in Section 6. The final section discusses the conclusion and directions.

\section{Literature Review}

In SCM, the BE means the phenomenon that demand enlargement as up to in SC, i.e., from the down demand to upstream (Lee et al. [1]). This phenomenon can lead to substantial problems that affect supply chain performance, such as superfluous inventory, and high costs for corrections (Lee et al. [10]). The BE has become one of the main obstacles affecting the efficiency of the SC.

Early papers concentrate on the existence of the BE and recognized its reasons (Forrester [11]; Lee et al. [1]; Lee et al. [10]). Over the next few years, many papers focused on quantifying the $\mathrm{BE}$ and identified corrective measures to reduce it in the simple two-level SC structure using different methods. Most of the relevant research quantified the BE using the statistical method under the autoregressive demand process, such as $\operatorname{AR}(1)$ which was examined by Lee et al. [1] and Lee et al. [12]; the ARMA demand of $(1,1)$ which was discussed in Alwan and others [13], Hausman [14], and Liu et al. [15]; the ARMA demand of $(p, q)$ which outlined in Gaalman and Disney [16] and Gaalman et al. [17]. Aforementioned papers discussed how the BE could be quantified in terms of different autoregressive demand processes. In addition, most research that examined the $\mathrm{BE}$ used different forecasting methods. Lee et al. [1] and Chen et al. [2] have gotten one conclusion to the study about the $\mathrm{BE}$ using the forecasting technique. Lee et al. [1] presented a quantitative model of the BE in two-level SC systems. Lee et al. [12] discussed that the demand information sharing could be very large, when the demand was related to the time. Chen et al. [2] quantified the BE in two-level SC systems, which includes a manufacturer and a retailer, by using the (MA) forecasting method. Based on that, Chen et al. [3] discussed the retailer's use the exponential smoothing (ES) method could cause the BE. Kim and Ryan [18] proposed an inventory model in which the retailer employs the ES method. Holland and Sodhi [19] posited the price fluctuation as causing the BE by using the ES method. Zhang $[8,20]$ analyzed the BE by using the minimum mean square error (MMSE) technique and compared the outcomes with the MA and ES technique. Hosoda and Disney [21] researched the $\mathrm{BE}$ and inventory variance using the MMSE method. Zhao and Xie [22] and Liu and Wang [9] discussed the BE in a multistage SC scenario with the ES technique. Some other forecasting methods have also been employed by $\mathrm{Ma}$, Wang, Huang, and $\mathrm{Xu}$ [23]. Ma et al. [24] offered insights into how quantitative the BE in twoparallel SC. Moreover, they analyzed the impact of MMSE method on the BE.

In addition to the statistical technique, the system control approach is widely used to study the BE in a twolevel SC. Holt et al. [25] pointed out that the HMMS model has balanced the relation between the retailer's order and supplier's order. This model provided the theoretical basis for many scholars to study the BE by using control theory. On the contrary, assuming that the retailer used $(S, S)$ ordering strategy, Blinder et al. [26, 27] reached a different conclusion. They highlighted how it could reduce the BE by using an inventory control strategy. Baganha et al. [28] discussed that it could effectively control the amplification of demand information by designing the appropriate inventory control strategy. Towill [29] studied the BE using discrete control techniques and $L$-transform methods. Huang et al. [30] pointed out that it could effectively reduce the BE by using the $H_{\propto}$ control theory. Disney et al. [31] derived an analytical model for the BE. Ingalls et al. [32] experimented with a control-based forecasting technique, and it could reduce the BE. Feng and $\mathrm{Ma}$ [33] measured the $\mathrm{BE}$ using inventory control theory and the $O-S$ feedback control method. Costantino et al. [34] evaluated an SPC forecasting system (SPC-FS) that utilized a control approach that was integrated with a set of simple decision rules to counteract the BE. Udenio et al. [35] built a system dynamics (SD) model to analyze the $\mathrm{BE}$ and dips observed in manufacturing industry. In addition to the above theoretical approach for quantifying the $\mathrm{BE}$ in a two-level SC, many scholars attempted to analyze the BE in two or multilevel SC by employing a simulation approach and empirical research (e.g., Costantino et al. [36]).

As is known to us, literatures analyze the BE under the case of the price sensitivity demand modeling. For example, Zhang and Burke [37] discussed the reason of the BE by considering price-sensitive demand streams. They pointed out that the demand autocorrelation, and price sensitivity were lead to the BE. Ma et al. [38] built the equation of the $\mathrm{BE}$ on product orders and inventory using the MMSE, ES, and MA forecasting techniques with the price sensitivity demand modeling. Gao et al. [39] investigated the difference in $\mathrm{BE}$ in online and offline SC, providing that the frequent price discounts impact the $\mathrm{BE}$. 
It is commonly accepted that there are three different types of flow in SC, i.e., demand flow, inventory flow, and cash flow. Moreover, a fluctuation in demand flow can cause a larger fluctuation in inventory flow. In the past several years, many scholars studied the problem of the impact of the $\mathrm{BE}$ on inventory flow and cash flow in two-level or multilevel SC. Ma et al. [38] gave the analytical mode of BE on product orders and inventory using the MMSE, ES, and MA forecasting techniques. Furthermore, Ma et al. [23] derived the analytical equations of the $\mathrm{BE}$ with and without information sharing. Tangsucheeva and Prabhu [40] theorized that the BE could lead to CFBE and analyzed the mathematical models for it. Goodarzi et al. [41] analyzed both the causes of an IBE effect and the effect of their interactions on CFBE based on a generalized "OUT" policy and CCC variance. Yuan et al. [42] have analyzed the influence of different techniques on the IBE considering the competition effect.

While many scholars researched the $\mathrm{BE}$, an increasing number of problems required further study, such as determining how to reduce the $\mathrm{BE}$ and how it may be quantified with respect to complex supply chain networks. The above studies have a lot of limitations: (1) the research concentrates on two-level or multilevel SC, and fewer papers evaluate two-parallel SC systems which include two suppliers and two retailers; (2) many scholars assume the trade of one type of product between two enterprises, and few consider the trade of two different types of products that are substitutable between them; (3) many papers discuss the influence of lead times, and other factors on the BE, while few study the impact of the above variables on the CFBE.

In contrast to previous studies, we provide an alternative perspective that controls the $\mathrm{BE}$, the IBE, and the $\mathrm{CFBE}$ using a statistical approach. The contribution is that: (1) the object involved two-product and two-parallel SC systems consisting of two suppliers and two retailers; (2) this study introduced the relevant factors in the model. Then, we obtained the equation of CFBE in two-product and twoparallel SC systems; and (3) we analyzed the condition that the competition effect and the market share could increase or reduce the CFBE. We have found that when two products were substitutable, the CFBE always existed and could not be reduced. Moreover, when two products shared the market, the CFBE could be reduced with the increasing of market share when the value of the correlation coefficient was lower than 0.5. Compared with previous research, this study found that the CFBE could be reduced, but only when the price cross-sensitivity coefficient was very small; otherwise, the CFBE always existed.

\section{Problem and Modelling}

Assuming that there are only two SCs in the market, and each consisted one supplier and one retailer, which are expressed as $S_{i}$ and $R_{i}(i=1,2)$, respectively. In addition, they trade one type product in each SC. The retailer was not only responsible for meeting the customer's needs but also ordered the product from the supplier. The supplier supplies the product to the retailer. Demand is transmitted downstream to upstream, inventory is transmitted upstream to downstream, and cash flow is transmitted downstream to upstream. The theoretical model can be seen in Figure 1. Two retailers (i.e., R1 and R2) share the same market, which not only depends on R1's market price but also on R2's price when the customer chooses R1's product. Assume that the probabilities of customers choosing R1's product and R2's product are considered as $\psi$ and $1-\psi$. In other words, the market shares of the two retailers are expressed as $\psi$ and $1-\psi$. Moreover, we assumed that there was a competition effect between the two SCs because there were two types of products that are substitutable. At the end of period $t$, both retailers placed an order, i.e., $q_{1, t}$ and $q_{2, t}$. Assuming that the lead time $l_{1}$ and $l_{2}$ were fixed, the order will be received at the beginning of period $t+l_{1}+1$ and $t+l_{2}+1$. Both retailers filled the consumer demand $d_{1, t}$ and $d_{2, t}$ and backlogged any excessive demand.

We know that the market price determines demand, and we referred to the price demand process. Two retailers faced the same demand process. Based on the above analysis, assuming the demand model of both retailers is that

$$
\begin{aligned}
& d_{1, t}=a_{1}-b_{11} p_{1, t}+b_{12} p_{2, t}+\varepsilon_{1, t}, \\
& d_{2, t}=a_{2}-b_{21} p_{2, t}+b_{22} p_{1, t}+\varepsilon_{2, t} .
\end{aligned}
$$

In equation (1), $a_{1}$ and $a_{2}$ express the potential market demand and are nonnegative constants, while $b_{11}$ and $b_{21}$ are price self-sensitivity coefficients which are nonnegative constants. In addition, $b_{12}$ and $b_{22}$ are price cross-sensitivity coefficients, $\varepsilon_{1, t}$ and $\varepsilon_{2, t}$ are demand shocks which are independent and identically normally distributed with a mean of zero and a variance of $\sigma_{1}^{2}$ and $\sigma_{2}^{2}$. It is that $b_{12}$ and $b_{22}$ are nonnegative because two types of products are substitutable.

Assume that the demand was a linear function of the price self-sensitivity coefficient and the price cross-sensitivity coefficient, that the price followed $\mathrm{AR}(1)$ process, and that two retailers shared the market.

In other words, prices $p_{1, t}$ and $p_{2, t}$ of the two retailers are

$$
\begin{aligned}
& p_{1, t}=\psi \mu_{1}+\rho_{1} p_{1, t-1}+\psi \eta_{1, t}, \\
& p_{2, t}=(1-\psi) \mu_{2}+\rho_{2} p_{2, t-1}+(1-\psi) \eta_{2, t} .
\end{aligned}
$$

In equation (2), $\psi$ and $1-\psi$ express the market share of $\mathrm{R} 1$ and R2, $\mu_{1}$ and $\mu_{2}$ are nonnegative constants, and $\rho_{1}$ and $\rho_{2}$ are the price autoregressive coefficients that satisfy $0<\rho_{1}<1,0<\rho_{2}<1$, while $\eta_{1, t}$ and $\eta_{2, t}$ are independent and identically normally distributed with a zero mean and a variance of $\delta_{1}^{2}$ and $\delta_{2}^{2}$, which express in the following covariance structure:

$$
\operatorname{Cov}\left(\eta_{1, t}, \eta_{2, t}\right)= \begin{cases}\delta_{12}^{2}, & t=t^{\prime} \\ 0, & t \neq t^{\prime}\end{cases}
$$

Based on the AR(1) model, in any period $t$, we obtain the expectation and variance of $p_{t}^{1}$ and $p_{t}^{2}$ : 


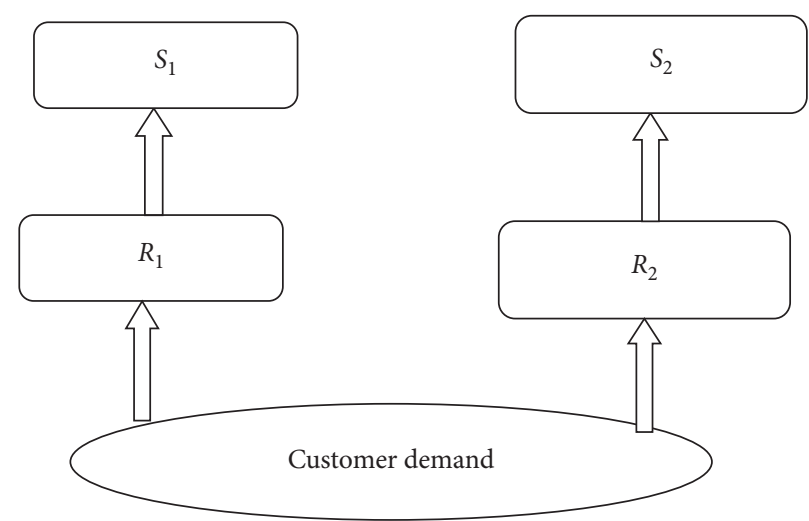

FIGURE 1: Theoretical model of the two-product and two-parallel supply chain systems.

$$
\begin{aligned}
& \mu_{1, p}=E\left(p_{1, t}\right)=\frac{\psi \mu_{1}}{1-\rho_{1}}, \quad \text { We can obtain the expectation and variance of } d_{1, t} \text { and } \\
& \mu_{2, p}=E\left(p_{2, t}\right)=\frac{(1-\psi) \mu_{2}}{1-\rho_{2}},\left(\delta_{1, p}\right)^{2}=\operatorname{Var}\left(p_{1, t}\right)=\frac{\psi^{2} \mu_{1}^{2}}{1-\rho_{1}^{2}} \\
& \left(\delta_{2, p}\right)^{2}=\operatorname{Var}\left(p_{2, t}\right)=\frac{(1-\psi)^{2} \mu_{2}^{2}}{1-\rho_{2}^{2}}
\end{aligned}
$$

$$
\begin{gathered}
\mu_{1, d}=E\left(d_{1, t}\right)=a_{1}-b_{11} \mu_{1, p}+b_{12} \mu_{2, p} \\
\mu_{2, d}=E\left(d_{2, t}\right)=a_{2}-b_{21} \mu_{2, p}+b_{22} \mu_{1, p} \\
\left(\delta_{1, d}\right)^{2}=\operatorname{Var}\left(d_{1, t}\right)=\sigma_{1}^{2}+b_{11}^{2}\left(\delta_{1, p}\right)^{2}+b_{12}^{2}\left(\delta_{2, p}\right)^{2}-\frac{2 b_{11} b_{12} \psi(1-\psi) \delta_{12}^{2}}{1-\rho_{1} \rho_{2}}, \\
\left(\delta_{2, d}\right)^{2}=\operatorname{Var}\left(d_{2, t}\right)=\sigma_{2}^{2}+b_{21}^{2}\left(\delta_{2, p}\right)^{2}+b_{22}^{2}\left(\delta_{1, p}\right)^{2}-\frac{2 b_{21} b_{22} \psi(1-\psi) \delta_{12}^{2}}{1-\rho_{1} \rho_{2}}
\end{gathered}
$$

Assume that two retailers use the "order-up-to" strategy. We propose that the lead time of R1 and R2 are fixed, and at the beginning of period $t$, the order quantity of two retailers are $q_{1, t}$ and $q_{2, t}$, such that $q_{1, t}$ and $q_{2, t}$ can be expressed relative to the demand $d_{1, t}$ and $d_{2, t}$ as follows:

$$
\begin{aligned}
& q_{1, t}=S_{1, t}-S_{1, t-1}+d_{1, t-1}, \\
& q_{2, t}=S_{2, t}-S_{2, t-1}+d_{2, t-1} .
\end{aligned}
$$

In equation (6), $S_{1, t}$ and $S_{2, t}$ express the highest inventory level for the two retailers. They are estimated from the observed demand such that

$$
\begin{aligned}
& S_{1, t}=\widehat{d}_{1, t}^{l_{1}}+z_{1} \hat{\xi}_{1, t}^{l_{1}}, \\
& S_{2, t}=\hat{d}_{2, t}^{l_{2}}+z_{2} \widehat{\xi}_{2 t}^{l_{2}} .
\end{aligned}
$$


In equation (7), $\hat{d}_{1, t}^{l_{1}}$ and $\hat{d}_{2, t}^{l_{2}}$ are two estimates of the mean lead time demands using the MA technique, $z_{1}$ and $z_{2}$ are two constants that express the goal to provide a desired service level, while $\vec{\xi}_{1, t}^{1}$ and $\widehat{\xi}_{2, t}^{2}$ are two estimates of the standard deviation of $l_{1}$ and $l_{2}$ period forecast errors of R1 and $\mathrm{R} 2$, respectively.
Lemma 1. Under the MA technique, the estimate of the standard deviation of the $l_{1}$ period forecast error of retailer$1 \vec{\xi}_{1, t}^{1}$ is a constant and

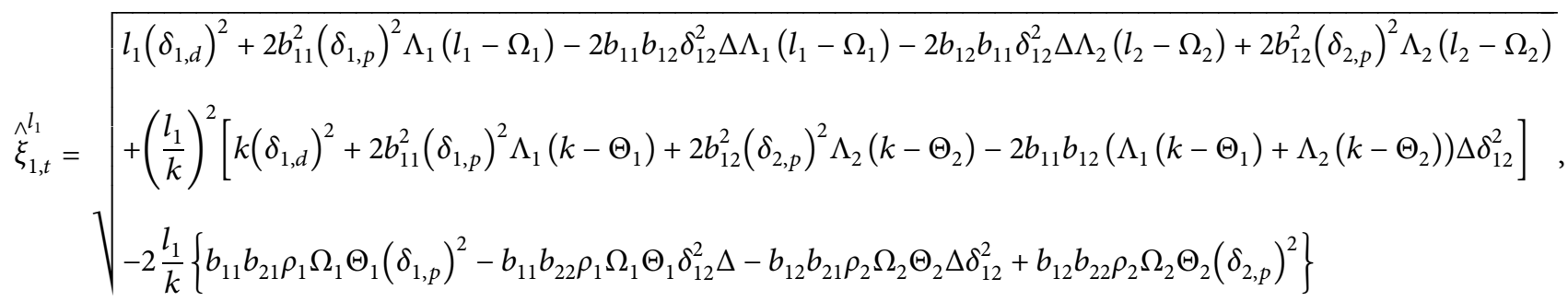

where, $\Lambda_{1}=\rho_{1} / 1-\rho_{1}, \Lambda_{2}=\rho_{2} / 1-\rho_{2}, \quad \Omega_{1}=1-\rho_{1}^{l_{1}} / 1-\rho_{1}$, $\Omega_{2}=1-\rho_{2}^{l_{2}} / 1-\rho_{2} \Theta_{1}=1-\rho_{1}^{k} / 1-\rho_{1}, \quad \Theta_{2}=1-\rho_{2}^{k} / 1-\rho_{2}$, and $\Delta=\psi(1-\psi) / 1-\rho_{1} \rho_{2}$.
Lemma 2. Under the MA method, the estimate of the standard deviation of the $l_{2}$ period forecast error of retailer$2 \hat{\xi}_{2, t}^{2}$ is a constant that

$$
\stackrel{\hat{\xi}}{2, t}=^{l_{2}}=\sqrt[\begin{array}{l}
l_{2}\left(\delta_{2, d}\right)^{2}+2 b_{21}^{2}\left(\delta_{2, p}\right)^{2} \Lambda_{2}\left(l_{2}-\Omega_{2}\right)-2 b_{21} b_{22} \delta_{12}^{2} \Delta \Lambda_{2}\left(l_{2}-\Omega_{2}\right)-2 b_{22} b_{21} \delta_{12}^{2} \Delta \Lambda_{1}\left(l_{1}-\Omega_{1}\right)+2 b_{22}^{2}\left(\delta_{1, p}\right)^{2} \Lambda_{1}\left(l_{1}-\Omega_{1}\right) \\
+\left(k\left(\delta_{2, d}\right)^{2}+2 b_{21}^{2}\left(\delta_{2, p}\right)^{2} \Lambda_{2}\left(k-\Theta_{2}\right)+2 b_{22}^{2}\left(\delta_{1, p}\right)^{2} \Lambda_{1}\left(k-\Theta_{1}\right)-2 b_{21} b_{22}\left(\Lambda_{1}\left(k-\Theta_{1}\right)+\Lambda_{2}\left(k-\Theta_{2}\right)\right) \Delta \delta_{12}^{2}\right]
\end{array}]{-2 \frac{l_{2}}{k}\left\{b_{21}^{2} \rho_{2} \Omega_{2} \Theta_{2}\left(\delta_{2, p}\right)^{2}-b_{21} b_{22} \delta_{12}^{2} \Delta \rho_{2} \Omega_{2} \Theta_{2}-b_{22} b_{21} \delta_{12}^{2} \Delta \rho_{1} \Omega_{1} \Theta_{1}+b_{22}^{2} \rho_{1}\left(\delta_{1, p}\right)^{2} \Omega_{1} \Theta_{1}\right\}},
$$

where, $\Lambda_{1}=\rho_{1} / 1-\rho_{1}, \Lambda_{2}=\rho_{2} / 1-\rho_{2}, \quad \Omega_{1}=1-\rho_{1}^{l_{1}} / 1-\rho_{1}$, $\Omega_{2}=1-\rho_{2}^{2} / 1-\rho_{2} \Theta_{1}=1-\rho_{1}^{k} / 1-\rho_{1}, \quad \Theta_{2}=1-\rho_{2}^{k} / 1-\rho_{2}$, and $\Delta=\psi(1-\psi) / 1-\rho_{1} \rho_{2}$.

Thus, the order quantity $q_{1, t}$ and $q_{2, t}$ for the two retailers can be written related to the estimates of the lead time demand $\hat{d}_{1, t}^{l_{1}}, \hat{d}_{2, \mathrm{t}}^{2}$ as

$$
\begin{aligned}
& q_{1, t}=\hat{d}_{1, t}^{l_{1}}-\widehat{d}_{1, t-1}^{l_{1}}+d_{1, t-1}, \\
& q_{2, t}=\hat{d}_{2, t}^{l_{2}}-\hat{d}_{2, t-1}^{l_{2}}+d_{2, t-1} .
\end{aligned}
$$

As we can see from equation (7), the inventory equation can consist of the highest inventory level $S_{t}$ and the demand forecasting value $\hat{d}_{t}$. In order to quantify the order quantity of the two retailers, we should estimate the demand forecasting value $\hat{d}_{t}^{l}$. We assume that the two retailers predict the lead time demand using the MA technique. Using the MA technique, $k$ is the moving average period, while $d_{1, t-i}$ and $d_{2, t-i}$ express the actual demand of the two retailers in period $t-i$. The lead time demand of $\mathrm{R} 1$ and $\mathrm{R} 2$ is

$$
\begin{aligned}
& \hat{d}_{1, t}^{l_{1}}=\frac{l_{1}}{k} \sum_{i=1}^{k} d_{1, t-i}, \\
& \hat{d}_{2, t}^{l_{2}}=\frac{l_{2}}{k} \sum_{i=1}^{k} d_{2, t-i} .
\end{aligned}
$$

\section{The Cash Flow Bullwhip Effect in Two-Product and Two-Parallel Supply Chain Systems}

Based on the price demand model and order-up-to strategy outlined above, we mainly developed the quantitative model of the $\mathrm{BE}$, the IBE, and the CFBE in two-product and twoparallel SC systems using the MA technique.

4.1. The Quantitative Model of the Bullwhip Effect. First, this section analyses the quantitative model of the BE. We combine equation (9) with equation (8), the order quantity of $\mathrm{R} 1$ and $\mathrm{R} 2$ is 


$$
\begin{aligned}
& q_{1, t}=\frac{l_{1}}{k} \sum_{i=1}^{k} d_{1, t-i}-\frac{l_{1}}{k} \sum_{i=1}^{k} d_{1, t-i-1}+d_{1, t-1}=\left(1+\frac{l_{1}}{k}\right) d_{1, t-1}-\frac{l_{1}}{k} d_{1, t-k-1}, \\
& q_{2, t}=\frac{l_{2}}{k} \sum_{i=1}^{k} d_{2, t-i}-\frac{l_{2}}{k} \sum_{i=1}^{k} d_{2, t-i-1}+d_{2, t-1}=\left(1+\frac{l_{2}}{k}\right) d_{2, t-1}-\frac{l_{2}}{k} d_{2, t-k-1} .
\end{aligned}
$$

The variance of the order quantity of $\mathrm{R} 1$ and $\mathrm{R} 2$ is

$$
\begin{aligned}
\operatorname{Var}\left(q_{1, t}\right)= & {\left[\left(1+\Lambda_{1}\right)^{2}+\Lambda_{1}^{2}\right]\left[\sigma_{1}^{2}+b_{11}^{2} \operatorname{Var}\left(p_{1, t}\right)+b_{12}^{2} \operatorname{Var}\left(p_{2, t}\right)-2 b_{11} b_{12} \delta_{12}^{2} \Pi\right]-2\left(1+\Lambda_{1}\right)\left(\Lambda_{1}\right)\left\{b_{11}^{2} \operatorname{Var}\left(p_{1, t}\right)+b_{12}^{2} \operatorname{Var}\left(p_{2, t}\right)\right.} \\
& \left.-b_{11} b_{12}\left(\rho_{1}^{k}+\rho_{2}^{k}\right) \delta_{12}^{2} \Pi\right\}
\end{aligned}
$$

and

$$
\begin{aligned}
\operatorname{Var}\left(q_{2, t}\right)= & {\left[\left(1+\Lambda_{2}\right)^{2}+\Lambda_{2}^{2}\right]\left[\sigma_{2}^{2}+b_{21}^{2} \operatorname{Var}\left(p_{2, t}\right)+b_{22}^{2} \operatorname{Var}\left(p_{1, t}\right)-2 b_{21} b_{22} \delta_{12}^{2} \Pi\right]-2\left(1+\Lambda_{2}\right)\left(\Lambda_{2}\right)\left\{b_{21}^{2} \rho_{2}^{k} \operatorname{Var}\left(p_{2, t}\right)\right.} \\
& \left.+b_{22}^{2} \rho_{1}^{k} \operatorname{Var}\left(p_{1, t}\right)-b_{21} b_{22}\left(\rho_{2}^{k}+\rho_{1}^{k}\right) \delta_{12}^{2} \Pi\right\},
\end{aligned}
$$

where $\Lambda_{1}=l_{1} / k, \Lambda_{2}=l_{2} / k$, and $\Pi=\psi(1-\psi) / 1-\rho_{1} \rho_{2}$.

and market share which uses the MA forecasting technique; the equation of the BE in two-parallel SC systems is

Theorem 1. Consider two-product and two-parallel SC systems in which retailers experience the competition effect

$\mathrm{BE}_{\text {retailer-1 }}=\frac{\operatorname{Var}\left(q_{1, t}\right)}{\operatorname{Var}\left(d_{1, t}\right)}$

$$
=1+\frac{\left[2 \Lambda_{1}^{2}+2 \Lambda_{1}\right]\left[\sigma_{1}^{2}+b_{11}^{2}\left(\delta_{1, p}\right)^{2}+b_{12}^{2}\left(\delta_{2, p}\right)^{2}-2 b_{11} b_{12} \delta_{12}^{2} \Delta\right]-2\left(1+\Lambda_{1}\right)\left(\Lambda_{1}\right)\left\{b_{11}^{2}\left(\delta_{1, p}\right)^{2}+b_{12}^{2}\left(\delta_{2, p}\right)^{2}-b_{11} b_{12}\left(\rho_{1}^{k}+\rho_{2}^{k}\right) \delta_{12}^{2} \Delta\right\}}{\left(\delta_{1, d}\right)^{2}}
$$

and

$$
\begin{aligned}
\mathrm{BE}_{\text {retailer-2 }} & =\frac{\operatorname{Var}\left(q_{2, t}\right)}{\operatorname{Var}\left(d_{2, t}\right)} \\
& =1+\frac{\left[2 \Lambda_{2}^{2}+2 \Lambda_{2}\right]\left[\sigma_{2}^{2}+b_{21}^{2}\left(\delta_{2, p}\right)^{2}+b_{22}^{2}\left(\delta_{1, p}\right)^{2}-2 b_{21} b_{22} \delta_{12}^{2} \Delta\right]-2\left(1+\Lambda_{2}\right)\left(\Lambda_{2}\right)\left\{b_{21}^{2} \rho_{2}^{k}\left(\delta_{2, p}\right)^{2}+b_{22}^{2} \rho_{1}^{k}\left(\delta_{1, p}\right)^{2}-b_{21} b_{22}\left(\rho_{2}^{k}+\rho_{1}^{k}\right) \delta_{12}^{2} \Delta\right\}}{\left(\delta_{2, d}\right)^{2}},
\end{aligned}
$$

where $\Lambda_{1}=l_{1} / k, \Lambda_{2}=l_{2} / k$, and $\Delta=\psi(1-\psi) / 1-\rho_{1} \rho_{2}$. The proof process can be seen in Appendix $A$.
Theorem 1 explains that the fluctuation in order quantity is greater than that of the quantity demanded. In other 
words, the BE exits in two-product and two-parallel SC systems.

4.2. The Quantitative Model of the Inventory Bullwhip Effect. In order to analyze the impact of the $\mathrm{BE}$ on inventory levels, we should calculate the variance of the inventory level of the both retailers at the period $t$ as $I_{1, t}$ and $I_{2, t}$, such that $I_{1, t}$ and $I_{2, t}$ are

$$
\begin{aligned}
& I_{1, t}=I_{1, t-1}+q_{1, t-l_{1}}-d_{1, t-1}, \\
& I_{2, t}=I_{2, t-1}+q_{2, t-l_{2}}-d_{2, t-1} .
\end{aligned}
$$

From equation (17), we obtain

$$
\begin{aligned}
& q_{1, t-l_{1}}=I_{1, t}-I_{1, t-1}+d_{1, t-1}, \\
& q_{2, t-l_{2}}=I_{2, t}-I_{2, t-1}+d_{2, t-1} .
\end{aligned}
$$

Vassian has proposed that $q_{1, t-l_{1}}$ and $q_{2, t-l_{2}}$ are

$$
\begin{aligned}
& q_{1, t-l_{1}}=\widehat{d}_{1, t}^{l_{1}}-\sum_{i=1}^{l_{1}-1} q_{1, t-i}-I_{1, t}, \\
& q_{2, t-l_{2}}=\widehat{d}_{2, t}^{l_{2}}-\sum_{i=1}^{l_{2}-1} q_{2, t-i}-I_{2, t} .
\end{aligned}
$$

By substituting equation (18) into equation (19), we obtain

$$
\begin{aligned}
& I_{1, t}=\widehat{\xi}_{1, t}^{l_{1}}=\widehat{d}_{1, t-l_{1}}^{l_{1}}-d_{1, t-l_{1}}^{l_{1}}, \\
& I_{2, t}=\widehat{\xi}_{2, t}^{l_{2}}=\hat{d}_{2, t-l_{2}}^{l_{2}}-d_{2, t-l_{2}}^{l_{2}} .
\end{aligned}
$$

Theorem 2. Consider two-product and two-parallel SC systems in which retailers experience a competition effect. When two retailers use the order-up-to strategy and the MA technique, the equation of the IBE in two SC systems is

$$
\begin{aligned}
\mathrm{IBE}_{\text {retailer- }}= & \frac{\operatorname{Var}\left(I_{1, t}\right)}{\operatorname{Var}\left(d_{1, t}\right)} \\
& \left\{\begin{array}{l}
l_{1}\left(\delta_{1, d}\right)^{2}+2 b_{11}^{2}\left(\delta_{1, p}\right)^{2} \Lambda_{1}\left(l_{1}-\Omega_{1}\right)-2 b_{11} b_{12} \delta_{12}^{2} \Delta \Lambda_{1}\left(l_{1}-\Omega_{1}\right)-2 b_{12} b_{11} \delta_{12}^{2} \Delta \Lambda_{2}\left(l_{2}-\Omega_{2}\right)+2 b_{12}^{2}\left(\delta_{2, p}\right)^{2} \Lambda_{2}\left(l_{2}-\Omega_{2}\right) \\
+\left(l_{1} / k\right)^{2}\left[k\left(\delta_{1, d}\right)^{2}+2 b_{11}^{2}\left(\delta_{1, p}\right)^{2} \Lambda_{1}\left(k-\Theta_{1}\right)+2 b_{12}^{2}\left(\delta_{2, p}\right)^{2} \Lambda_{2}\left(k-\Theta_{2}\right)-2 b_{11} b_{12}\left(\Lambda_{1}\left(k-\Theta_{1}\right)+\Lambda_{2}\left(k-\Theta_{2}\right)\right) \Delta \delta_{12}^{2}\right] \\
-2 l_{1} / k\left\{b_{11} b_{21} \rho_{1} \Omega_{1} \Theta_{1}\left(\delta_{1, p}\right)^{2}-b_{11} b_{22} \rho_{1} \Omega_{1} \Theta_{1} \delta_{12}^{2} \Delta-b_{12} b_{21} \rho_{2} \Omega_{2} \Theta_{2} \Delta \delta_{12}^{2}+b_{12} b_{22} \rho_{2} \Omega_{2} \Theta_{2}\left(\delta_{2, p}\right)^{2}\right\}
\end{array}\right. \\
= & \left(\delta_{1, d}\right)^{2}
\end{aligned}
$$

$$
\begin{aligned}
\operatorname{IBE}_{\text {retailer-2 }}= & \frac{\operatorname{Var}\left(I_{2, t}\right)}{\operatorname{Var}\left(d_{2, t}\right)} \\
& =\frac{\left\{\begin{array}{c}
l_{2}\left(\delta_{2, d}\right)^{2}+2 b_{21}^{2}\left(\delta_{2, p}\right)^{2} \Lambda_{2}\left(l_{2}-\Omega_{2}\right)-2 b_{21} b_{22} \delta_{12}^{2} \Delta \Lambda_{2}\left(l_{2}-\Omega_{2}\right)-2 b_{22} b_{21} \delta_{12}^{2} \Delta \Lambda_{1}\left(l_{1}-\Omega_{1}\right)+2 b_{22}^{2}\left(\delta_{1, p}\right)^{2} \Lambda_{1}\left(l_{1}-\Omega_{1}\right) \\
+\left(l_{2} / k\right)^{2}\left[k\left(\delta_{2, d}\right)^{2}+2 b_{21}^{2}\left(\delta_{2, p}\right)^{2} \Lambda_{2}\left(k-\Theta_{2}\right)+2 b_{22}^{2}\left(\delta_{1, p}\right)^{2} \Lambda_{1}\left(k-\Theta_{1}\right)-2 b_{21} b_{22}\left(\Lambda_{1}\left(k-\Theta_{1}\right)+\Lambda_{2}\left(k-\Theta_{2}\right)\right) \Delta \delta_{12}^{2}\right] \\
-2 l_{2} / k\left\{b_{21}^{2} \rho_{2} \Omega_{2} \Theta_{2}\left(\delta_{2, p}\right)^{2}-b_{21} b_{22} \delta_{12}^{2} \Delta \rho_{2} \Omega_{2} \Theta_{2}-b_{22} b_{21} \delta_{12}^{2} \Delta \rho_{1} \Omega_{1} \Theta_{1}+b_{22}^{2} \rho_{1}\left(\delta_{1, p}\right)^{2} \Omega_{1} \Theta_{1}\right\}
\end{array}\right.}{\left(\delta_{2, d}\right)^{2}},
\end{aligned}
$$

where $\quad \Lambda_{1}=\rho_{1} / 1-\rho_{1} \Lambda_{2}=\rho_{2} / 1-\rho_{2} \Omega_{1}=1-\rho_{1}^{l_{1}} / 1-\rho_{1}$, $\Omega_{2}=1-\rho_{2}^{l_{2}} / 1-\rho_{2} \Theta_{1}=1-\rho_{1}^{k} / 1-\rho_{1} \Theta_{2}=1-\rho_{2}^{k} / 1-\rho_{2}$, and $\Delta=\psi(1-\psi) / 1-\rho_{1} \rho_{2}$. The proof process can be seen in Appendix A.
Theorem 2 explains that the fluctuation in inventory quantity is greater than that of the quantity demanded. In other words, the IBE exits in two-product and two-parallel SC systems. 
4.3. Quantitative Model of Cash Flow Bullwhip Effect. We can see that the increase in the BE amplifies the variability of the inventory level, which results in the increase in the cash conversion cycle (CCC). Thus, CCC can be defined as follows:

$\mathrm{CCC}=\frac{\text { average inventory }}{\mathrm{COGS} / 365}(\mathrm{DIO})+\frac{\text { average } \text { account receivable }}{\text { revenue } / 365}(\mathrm{DSO})-\frac{\text { average account payable }}{\mathrm{COGS} / 365}(\mathrm{DPO})$.

In this section, the method proposed by Tangsucheeva and Prabhu is considered, and their cash flow assumption is taken into account. Accordingly, each of the three elements of CCC (i.e., inventory quantity, order quantity, and demand) is

$$
\begin{aligned}
\mathrm{DIO} & =365\left(\frac{S}{C}\right)\left(\frac{I}{D}\right), \\
\mathrm{DSO} & =365\left(\frac{I}{D}\right), \quad D>I, \\
\mathrm{DPO} & =365\left(\frac{q}{D}\right) .
\end{aligned}
$$

Based on the above equations, we can calculate CCC as follows:

$$
\mathrm{CCC}=365\left(\frac{S}{C}\right)\left(\frac{I}{D}\right)+365\left(\frac{I}{D}\right)-365\left(\frac{q}{D}\right),
$$

where $I$ expresses the average inventory level, $C$ expresses the unit cost, $D$ expresses the average demand, $S$ expresses the sale price per unit, and $q$ expresses the average order quantity.

The equation of the cash flow bullwhip effect (the definition of the cash flow bullwhip effect can be seen in the paper written by Tangsucheeva and Prabhu) such that

$$
\begin{aligned}
\text { CFBE }= & \frac{\operatorname{Var}(C C C)}{\operatorname{Var}(D)}=\frac{365^{2}}{E(D)^{2}} \times\left(\frac{S}{C}\right)^{2} \times \frac{\operatorname{Var}(I)}{\operatorname{Var}(D)}+\frac{365^{2}}{E(D)^{2}} \times\left(\frac{S}{C}\right)^{2} \times\left(\frac{E(I)}{E(D)}\right)^{2}+\frac{365^{2}}{E(D)^{2}} \times \frac{\operatorname{Var}(I)}{\operatorname{Var}(D)} \\
& +\frac{365^{2}}{E(D)^{2}} \times\left(\frac{E(I)}{E(D)}\right)^{2}+\frac{365^{2}}{E(D)^{2}} \times\left[\frac{\operatorname{Var}(q)}{\operatorname{Var}(D)}\right]+\frac{365^{2}}{E(D)^{2}} \times\left(\frac{E(q)}{E(D)}\right)^{2} .
\end{aligned}
$$

Theorem 3. Consider two-product and two-parallel SC systems in which retailers experience a competition effect and market share. When two retailers use the order-up-to inventory strategy and the MA technique, the equation of the CFBE in two SC systems is

$$
\begin{aligned}
\mathrm{CFBE}_{\text {retailer-1 }}= & \frac{\operatorname{Var}(\mathrm{CCC})}{\operatorname{Var}\left(d_{1, t}\right)}=\frac{365^{2}}{E\left(d_{1, t}\right)^{2}} \times\left(\frac{S_{1}}{C_{1}}\right)^{2} \mathrm{IBE}_{\text {retailer-1 }}+\frac{365^{2}}{E\left(d_{1, t}\right)^{2}} \times\left(\frac{S_{1}}{C_{1}}\right)^{2} \times\left(\frac{E\left(I_{1, t}\right)}{E\left(d_{1, t}\right)}\right)^{2}+\frac{365^{2}}{E\left(d_{1, t}\right)^{2}} \mathrm{IBE}_{\text {retailer-1 }} \\
& +\frac{365^{2}}{E\left(d_{1, t}\right)^{2}} \times\left(\frac{E\left(I_{1, t}\right)}{E\left(d_{1, t}\right)}\right)^{2}+\frac{365^{2}}{E\left(d_{1, t}\right)^{2}} B E_{\text {retailer-1 }}+\frac{365^{2}}{E\left(d_{1, t}\right)^{2}} \times\left(\frac{E\left(q_{1, t}\right)}{E\left(d_{1, t}\right)}\right)^{2}
\end{aligned}
$$

and

$$
\begin{aligned}
\mathrm{CFBE}_{\text {retailer-2 }}= & \frac{\operatorname{Var}(\mathrm{CCC})}{\operatorname{Var}\left(d_{2, t}\right)}=\frac{365^{2}}{E\left(d_{2, t}\right)^{2}} \times\left(\frac{S_{2}}{C_{2}}\right)^{2} \mathrm{IBE}_{\text {retailer-2 }}+\frac{365^{2}}{E\left(d_{2, t}\right)^{2}} \times\left(\frac{S_{2}}{C_{2}}\right)^{2} \times\left(\frac{E\left(I_{2, t}\right)}{E\left(d_{2, t}\right)}\right)^{2}+\frac{365^{2}}{E\left(d_{2, t}\right)^{2}} \mathrm{IBE}_{\text {retailer-2 }} \\
& +\frac{365^{2}}{E\left(d_{2, t}\right)^{2}} \times\left(\frac{E\left(I_{2, t}\right)}{E\left(d_{2, t}\right)}\right)^{2}+\frac{365^{2}}{E\left(d_{2, t}\right)^{2}} B E_{\text {retailer-2 }}+\frac{365^{2}}{E\left(d_{2, t}\right)^{2}} \times\left(\frac{E\left(q_{2, t}\right)}{E\left(d_{2, t}\right)}\right)^{2} .
\end{aligned}
$$


Theorem 3 explains that the fluctuation in cash flow is greater than that of the quantity demanded. In other words, the CFBE exits in two-product and two-parallel SC systems.

\section{Analysis of the Cash Flow Bullwhip Effect Using Different Demand Models}

We can develop the equation of the cash flow bullwhip effect from Theorem 3, which has the same structure involving two retailers. We analyze the impact of the price self-sensitivity coefficient, the price cross-sensitivity coefficient, and the market share on the cash flow bullwhip effect in respect to $\mathrm{R} 1$. We divide the above factors into the single scenario and the compounding scenario. The result can be seen as follows.

\subsection{The Single Scenario of the Cash Flow Bullwhip Effect}

Scenario 1. We assume that $b_{11} \neq 0, b_{21} \neq 0, b_{12}=b_{22}$ $=\delta_{12}^{2}=\psi=0$, and the quantitative model of the CFBE for R1 can be expressed as follows:

$$
\begin{aligned}
& \left(\left(S_{1} / C_{1}\right)^{2}+1\right) \times 365^{2} / E\left(d_{1, t}\right)^{2} \times\left\{l_{1}\left(\delta_{1, d}\right)^{2}+2 b_{11}^{2}\left(\delta_{1, p}\right)^{2} \Lambda_{1}\left(l_{1}-\Omega_{1}\right)+k\left(\delta_{1, d}\right)^{2}\left(l_{1} / k\right)^{2}-2 l_{1} / k\left\{b_{11} b_{21} \rho_{1} \Omega_{1} \Theta_{1}\left(\delta_{1, p}\right)^{2}\right\}\right\} \\
\mathrm{CFBE}_{\text {retailer-1 }}^{\text {self }}= & +365^{2} / E\left(d_{1, t}\right)^{2} \times\left(E\left(q_{1, t}\right) / E\left(d_{1, t}\right)\right)^{2} \\
& +\frac{365^{2}}{E\left(d_{1, t}\right)^{2}} \times\left\{1+\frac{\sigma_{1}^{2}\left[2 \Lambda_{1}^{2}+2 \Lambda_{1}\right]}{\left(\delta_{1, d}\right)^{2}}\right\}+\frac{365^{2}}{E\left(d_{1, t}\right)^{2}} \times\left(\frac{S_{1}}{C_{1}}\right)^{2} \times\left(\frac{E\left(I_{1, t}\right)}{E\left(d_{1, t}\right)}\right)^{2}+\frac{365^{2}}{E\left(d_{1, t}\right)^{2}} \times\left(\frac{E\left(I_{1, t}\right)}{E\left(d_{1, t}\right)}\right)^{2} .
\end{aligned}
$$

Scenario 2. We assume that $b_{12} \neq 0, b_{22} \neq 0, b_{11}=b_{21}$ $=\delta_{12}^{2}=\psi=0$, and the quantitative model of the CFBE for R1 can be expressed as follows:

$$
\begin{aligned}
\mathrm{CFBE}_{\text {retailer }-1}^{\text {cross }}= & \frac{\left(\left(S_{1} / C_{1}\right)^{2}+1\right) \times 365^{2} / E\left(d_{1, t}\right)^{2} \times\left\{\begin{array}{c}
l_{1}\left(\delta_{1, d}\right)^{2}+2 b_{12}^{2}\left(\delta_{2, p}\right)^{2} \Lambda_{2}\left(l_{2}-\Omega_{2}\right)+\left(l_{1} / k\right)^{2}\left[k\left(\delta_{1, d}\right)^{2}+2 b_{12}^{2}\left(\delta_{2, p}\right)^{2} \Lambda_{2}\left(k-\Theta_{2}\right)\right] \\
-2\left(l_{1} / k\right)\left\{b_{12} b_{22} \rho_{2} \Omega_{2} \Theta_{2}\left(\delta_{2, p}\right)^{2}\right\}
\end{array}\right.}{\left(\delta_{1, d}\right)^{2}} \\
& +\frac{365^{2}}{E\left(d_{1, t}\right)^{2}} \times\left\{1+\frac{\sigma_{1}^{2}\left[2 \Lambda_{1}^{2}+2 \Lambda_{1}\right]}{\left(\delta_{1, d}\right)^{2}}\right\}+\frac{365^{2}}{E\left(d_{1, t}\right)^{2}} \times\left(\frac{S_{1}}{C_{1}}\right)^{2} \times\left(\frac{E\left(I_{1, t}\right)}{E\left(d_{1, t}\right)}\right)^{2}+\frac{365^{2}}{E\left(d_{1, t}\right)^{2}} \times\left(\frac{E\left(I_{1, t}\right)}{E\left(d_{1, t}\right)}\right)^{2}+\frac{365^{2}}{E\left(d_{1, t}\right)^{2}} \times\left(\frac{E\left(q_{1, t}\right)}{E\left(d_{1, t}\right)}\right)^{2} .
\end{aligned}
$$

Scenario 3. We assume that $\psi \neq 0, b_{11}=b_{12}=b_{21}$ $=b_{22}=\delta_{12}^{2}=0$, and the quantitative model of the CFBE for $\mathrm{R} 1$ can be expressed as follows:

$$
\begin{aligned}
\mathrm{CFBE}_{\text {retailer-1 }}^{\text {market }}= & \frac{\left(\left(S_{1} / C_{1}\right)^{2}+1\right) \times 365^{2} / E\left(d_{1, t}\right)^{2} \times\left\{l_{1}\left(\delta_{1, d}\right)^{2}+\left(l_{1} / k\right)^{2}\left[k\left(\delta_{1, d}\right)^{2}\right]\right\}}{\left(\delta_{1, d}\right)^{2}} \\
& +\frac{365^{2}}{E\left(d_{1, t}\right)^{2}} \times\left\{1+\frac{\sigma_{1}^{2}\left[2 \Lambda_{1}^{2}+2 \Lambda_{1}\right]}{\left(\delta_{1, d}\right)^{2}}\right\}+\frac{365^{2}}{E\left(d_{1, t}\right)^{2}} \times\left(\frac{S_{1}}{C_{1}}\right)^{2} \times\left(\frac{E\left(I_{1, t}\right)}{E\left(d_{1, t}\right)}\right)^{2} \\
& +\frac{365^{2}}{E\left(d_{1, t}\right)^{2}} \times\left(\frac{E\left(I_{1, t}\right)}{E\left(d_{1, t}\right)}\right)^{2}+\frac{365^{2}}{E\left(d_{1, t}\right)^{2}} \times\left(\frac{E\left(q_{1, t}\right)}{E\left(d_{1, t}\right)}\right)^{2} .
\end{aligned}
$$


Scenario 4. We assume that $b_{11} \neq 0, b_{12} \neq 0, b_{21} \neq 0, b_{22} \neq 0$, $\delta_{12}^{2}=\psi=0$, and the quantitative model of the CFBE for R1 can be expressed as follows:

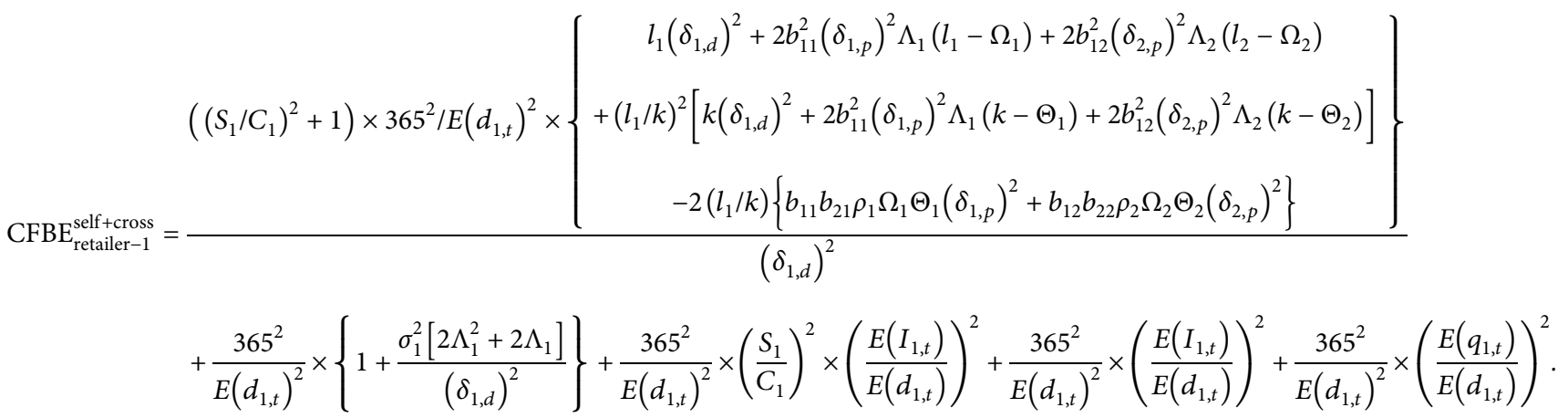

Scenario 5. We assume that $b_{11} \neq 0, b_{21} \neq 0, \psi \neq 0, b_{12}=b_{22}$ $=\delta_{12}^{2}=0$, and the quantitative model of the CFBE for R1 can be expressed as follows:

$$
\begin{aligned}
\mathrm{CFBE}_{\text {retailer-1 }}^{\text {self }+ \text { market }}= & \frac{\left(\left(S_{1} / C_{1}\right)^{2}+1\right) \times 365^{2} / E\left(d_{1, t}\right)^{2} \times\left\{\begin{array}{c}
l_{1}\left(\delta_{1, d}\right)^{2}+2 b_{11}^{2}\left(\delta_{1, p}\right)^{2} \Lambda_{1}\left(l_{1}-\Omega_{1}\right)+\left(l_{1} / k\right)^{2}\left[k\left(\delta_{1, d}\right)^{2}+2 b_{11}^{2}\left(\delta_{1, p}\right)^{2} \Lambda_{1}\left(k-\Theta_{1}\right)\right] \\
-2\left(l_{1} / k\right)\left\{b_{11} b_{21} \rho_{1} \Omega_{1} \Theta_{1}\left(\delta_{1, p}\right)^{2}\right\}
\end{array}\right.}{\left(\delta_{1, d}\right)^{2}} \\
& +\frac{365^{2}}{E\left(d_{1, t}\right)^{2}} \times\left\{1+\frac{\sigma_{1}^{2}\left[2 \Lambda_{1}^{2}+2 \Lambda_{1}\right]}{\left(\delta_{1, d}\right)^{2}}\right\}+\frac{365^{2}}{E\left(d_{1, t}\right)^{2}} \times\left(\frac{S_{1}}{C_{1}}\right)^{2} \times\left(\frac{E\left(I_{1, t}\right)}{E\left(d_{1, t}\right)}\right)^{2}+\frac{365^{2}}{E\left(d_{1, t}\right)^{2}} \times\left(\frac{E\left(I_{1, t}\right)}{E\left(d_{1, t}\right)}\right)^{2}+\frac{365^{2}}{E\left(d_{1, t}\right)^{2}} \times\left(\frac{E\left(q_{1, t}\right)}{E\left(d_{1, t}\right)}\right)^{2} .
\end{aligned}
$$

Scenario 6. We assume that $b_{12} \neq 0, b_{22} \neq 0, \psi \neq 0, b_{11}=b_{21}$ $=\delta_{12}^{2}=0$, and the quantitative model of the CFBE for R1 can be expressed as follows:

$$
\begin{aligned}
\mathrm{CFBE}_{\text {retailer-1 }}^{\text {crossarket }}= & \frac{\left(\left(S_{1} / C_{1}\right)^{2}+1\right) \times 365^{2} / E\left(d_{1, t}\right)^{2} \times\left\{\begin{array}{c}
l_{1}\left(\delta_{1, d}\right)^{2}+2 b_{12}^{2}\left(\delta_{2, p}\right)^{2} \Lambda_{2}\left(l_{2}-\Omega_{2}\right)+\left(l_{1} / k\right)^{2}\left[k\left(\delta_{1, d}\right)^{2}+2 b_{12}^{2}\left(\delta_{2, p}\right)^{2} \Lambda_{2}\left(k-\Theta_{2}\right)\right] \\
-2\left(l_{1} / k\right)\left\{b_{12} b_{22} \rho_{2} \Omega_{2} \Theta_{2}\left(\delta_{2, p}\right)^{2}\right\}
\end{array}\right.}{\left(\delta_{1, d}\right)^{2}} \\
& +\frac{365^{2}}{E\left(d_{1, t}\right)^{2}} \times\left\{1+\frac{\sigma_{1}^{2}\left[2 \Lambda_{1}^{2}+2 \Lambda_{1}\right]}{\left(\delta_{1, d}\right)^{2}}\right\}+\frac{365^{2}}{E\left(d_{1, t}\right)^{2}} \times\left(\frac{S_{1}}{C_{1}}\right)^{2} \times\left(\frac{E\left(I_{1, t}\right)}{E\left(d_{1, t}\right)}\right)^{2}+\frac{365^{2}}{E\left(d_{1, t}\right)^{2}} \times\left(\frac{E\left(I_{1, t}\right)}{E\left(d_{1, t}\right)}\right)^{2}+\frac{365^{2}}{E\left(d_{1, t}\right)^{2}} \times\left(\frac{E\left(q_{1, t}\right)}{E\left(d_{1, t}\right)}\right)^{2} .
\end{aligned}
$$

Scenario 7 . We assume that $b_{11} \neq 0, b_{12} \neq 0, b_{21} \neq 0, b_{22}$ $\neq 0, \psi \neq 0, \delta_{12}^{2}=0$, and the quantitative model of the CFBE in R1 can be expressed as follows: 


$$
\begin{aligned}
\mathrm{CFBE}_{\text {retailer-1 }}^{\text {self crosstmarket }}= & \frac{\left(\left(S_{1} / C_{1}\right)^{2}+1\right) \times 365^{2} / E\left(d_{1, t}\right)^{2} \times\left\{\begin{array}{c}
l_{1}\left(\delta_{1, d}\right)^{2}+2 b_{11}^{2}\left(\delta_{1, p}\right)^{2} \Lambda_{1}\left(l_{1}-\Omega_{1}\right)+2 b_{12}^{2}\left(\delta_{2, p}\right)^{2} \Lambda_{2}\left(l_{2}-\Omega_{2}\right) \\
+\left(l_{1} / k\right)^{2}\left[k\left(\delta_{1, d}\right)^{2}+2 b_{11}^{2}\left(\delta_{1, p}\right)^{2} \Lambda_{1}\left(k-\Theta_{1}\right)+2 b_{12}^{2}\left(\delta_{2, p}\right)^{2} \Lambda_{2}\left(k-\Theta_{2}\right)\right] \\
-2\left(l_{1} / k\right)\left\{b_{11} b_{21} \rho_{1} \Omega_{1} \Theta_{1}\left(\delta_{1, p}\right)^{2}+b_{12} b_{22} \rho_{2} \Omega_{2} \Theta_{2}\left(\delta_{2, p}\right)^{2}\right\}
\end{array}\right\}}{\left(\delta_{1, d}\right)^{2}} \\
& +\frac{365^{2}}{E\left(d_{1, t}\right)^{2}} \times\left\{1+\frac{\sigma_{1}^{2}\left[2 \Lambda_{1}^{2}+2 \Lambda_{1}\right]}{\left(\delta_{1, d}\right)^{2}}\right\}+\frac{365^{2}}{E\left(d_{1, t}\right)^{2}} \times\left(\frac{S_{1}}{C_{1}}\right)^{2} \times\left(\frac{E\left(I_{1, t}\right)}{E\left(d_{1, t}\right)}\right)^{2}+\frac{365^{2}}{E\left(d_{1, t}\right)^{2}} \times\left(\frac{E\left(I_{1, t}\right)}{E\left(d_{1, t}\right)}\right)^{2}+\frac{365^{2}}{E\left(d_{1, t}\right)^{2}} \times\left(\frac{E\left(q_{1, t}\right)}{E\left(d_{1, t}\right)}\right)^{2} .
\end{aligned}
$$

Scenario 8. We assume that $b_{11} \neq 0, b_{12} \neq 0, b_{21} \neq 0, b_{22}$ $\neq 0, \psi \neq 0, \delta_{12}^{2} \neq 0$, and the quantitative model of the CFBE in $\mathrm{R} 1$ can be expressed as follows:

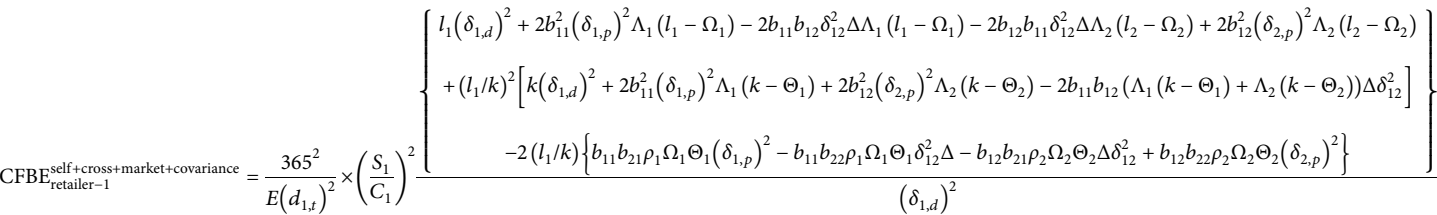

$$
\begin{aligned}
& +\frac{365^{2}}{E\left(d_{1, t}\right)^{2}} \times\left(\frac{S_{1}}{C_{1}}\right)^{2} \times\left(\frac{E\left(I_{1, t}\right)}{E\left(d_{1, t}\right)}\right)^{2}+\frac{365^{2}}{E\left(d_{1, t}\right)^{2}} \frac{\left\{\begin{array}{c}
l_{1}\left(\delta_{1, d}\right)^{2}+2 b_{11}^{2}\left(\delta_{1, p}\right)^{2} \Lambda_{1}\left(l_{1}-\Omega_{1}\right)-2 b_{11} b_{12} \delta_{12}^{2} \Delta \Lambda_{1}\left(l_{1}-\Omega_{1}\right)-2 b_{12} b_{11} \delta_{12}^{2} \Delta \Lambda_{2}\left(l_{2}-\Omega_{2}\right)+2 b_{12}^{2}\left(\delta_{2, p}\right)^{2} \Lambda_{2}\left(l_{2}-\Omega_{2}\right) \\
+\left(l_{1} / k\right)^{2}\left[k\left(\delta_{1, d}\right)^{2}+2 b_{11}^{2}\left(\delta_{1, p}\right)^{2} \Lambda_{1}\left(k-\Theta_{1}\right)+2 b_{12}^{2}\left(\delta_{2, p}\right)^{2} \Lambda_{2}\left(k-\Theta_{2}\right)-2 b_{11} b_{12}\left(\Lambda_{1}\left(k-\Theta_{1}\right)+\Lambda_{2}\left(k-\Theta_{2}\right)\right) \Delta \delta_{12}^{2}\right] \\
-2\left(l_{1} / k\right)\left\{b_{11} b_{21} \rho_{1} \Omega_{1} \Theta_{1}\left(\delta_{1, p}\right)^{2}-b_{11} b_{22} \rho_{1} \Omega_{1} \Theta_{1} \delta_{12}^{2} \Delta-b_{12} b_{21} \rho_{2} \Omega_{2} \Theta_{2} \Delta \delta_{12}^{2}+b_{12} b_{22} \rho_{2} \Omega_{2} \Theta_{2}\left(\delta_{2, p}\right)^{2}\right\}
\end{array}\right\}}{\left(\delta_{1, d}\right)^{2}} \\
& +\frac{365^{2}}{E\left(d_{1, t}\right)^{2}} \times\left(\frac{E\left(I_{1, t}\right)}{E\left(d_{1, t}\right)}\right)^{2}+\frac{365^{2}}{E\left(d_{1, t}\right)^{2}}\left\{1+\frac{\left[2 \Lambda_{1}^{2}+2 \Lambda_{1}\right]\left[\sigma_{1}^{2}+b_{11}^{2}\left(\delta_{1, p}\right)^{2}+b_{12}^{2}\left(\delta_{2, p}\right)^{2}-2 b_{11} b_{12} \delta_{12}^{2} \Delta\right]-2\left(1+\Lambda_{1}\right)\left(\Lambda_{1}\right)\left\{b_{11}^{2}\left(\delta_{1, p}\right)^{2}+b_{12}^{2}\left(\delta_{2, p}\right)^{2}-b_{11} b_{12}\left(\rho_{1}^{k}+p_{2}^{k}\right) \delta_{12}^{2} \Delta\right\}}{\left(\delta_{1, d}\right)^{2}}\right\} \\
& +\frac{365^{2}}{E\left(d_{1, t}\right)^{2}} \times\left(\frac{E\left(q_{1, t}\right)}{E\left(d_{1, t}\right)}\right)^{2} \text {. }
\end{aligned}
$$

Proposition for "Retailer 1" is as follows:

(i) If there is only the price self-sensitivity coefficient, the CFBE for "Retailer 1" always exists. If there is only the price cross-sensitivity coefficient, the CFBE for "Retailer 1" always exists. If there is only the market share, the CFBE for "Retailer 1" also always exists.

(ii) If only the market share is introduced incrementally to "Scenario 1," the CFBE always exists. However, compared with "Scenario 1," the CFBE can be increased when the following condition holds: $b_{11}^{2}\left(\delta_{1, p}\right)^{2}$ $\Lambda_{1}\left(k-\Theta_{1}\right)>0$. Otherwise, the CFBE will be decreased.

(iii) If only the price cross-sensitivity coefficient is introduced incrementally to "Scenario 1," the CFBE always exists. However, compared with "Scenario 1 ," the CFBE can be increased when it satisfies the condition: $b_{12} b_{22} \rho_{2} \Omega_{2} \Theta_{2} \quad\left(\delta_{2, p}\right)^{2}+b_{12}^{2}\left(\delta_{2, p}\right)^{2} \Lambda_{2}$ $\left(l_{2}-\Omega_{2}\right) k-b_{12}^{2}\left(\delta_{2, p}\right)^{2} \Lambda_{2}\left(k-\Theta_{2}\right)>b_{11}^{2}\left(\delta_{1, p}\right)^{2} \Lambda_{1}(k$ $\left.-\Theta_{1}\right)$. (iv) If the price cross-sensitivity coefficient and the market share are introduced incrementally to "Scenario 1," the cash flow bullwhip effect always exists. The CFBE can be increased when the following condition holds: $\left(b_{11}^{2}+b_{12}^{2}\right)\left(\delta_{1, p}\right)^{2} \Lambda_{1}(k-$ $\left.\Theta_{1}\right)>b_{12} b_{22} \rho_{2} \Omega_{2} \Theta_{2}\left(\delta_{2, p}\right)^{2}+b_{12}^{2}\left(\delta_{2, p}\right)^{2} \Lambda_{2}\left(l_{2}-\Omega_{2}\right)$ $k-b_{12}^{2}\left(\delta_{2, p}\right)^{2} \Lambda_{2}\left(k-\Theta_{2}\right)$. Otherwise, the CFBE will be decreased.

(v) If the price cross-sensitivity coefficient, the market share, and the covariance are introduced incrementally to "Scenario 1," the CFBE always exists. The CFBE can be increased when the following condition holds: $\left\{1+2\left(1+\Lambda_{1}\right)\right.$ $\left.\left(\Lambda_{1}\right)\left\{b_{11} b_{12}\left(\rho_{1}^{k}+\rho_{2}^{k}\right) \delta_{12}^{2} \Delta\right\} /\left(\delta_{1, d}\right)^{2}\right\}>\left\{\left(S_{1} / C_{1}\right)^{2}+1\right\}$ $\left\{\begin{array}{c}2 b_{11} b_{12} \delta_{12}^{2} \Delta \Lambda_{1}\left(l_{1}-\Omega_{1}\right)+2 b_{12} b_{11} \delta_{12}^{2} \Delta \Lambda_{2}\left(l_{2}-\Omega_{2}\right) \\ +\left(l_{1} / k\right)^{2}\left[2 b_{11} b_{12}\left(\Lambda_{1}\left(k-\Theta_{1}\right)-\Lambda_{2}\left(k-\Theta_{2}\right)\right) \Delta \delta_{12}^{2}\right] \\ -2\left(l_{1} / k\right)\left\{b_{11} b_{22} \rho_{1} \Omega_{1} \Theta_{1} \delta_{12}^{2} \Delta+b_{12} b_{21} \rho_{2} \Omega_{2} \Theta_{2} \Delta \delta_{12}^{2}\right\}\end{array}\right\}$ $/\left(\delta_{1, d}\right)^{2}$ Otherwise, the CFBE will be decreased. 
(vi) Comparing "Scenario 5" with "Scenario 7," based on the interaction demand model and the introduction of only the market share into "Scenario 5," the CFBE shows no change. In this case, the market share has no impact on reducing the CFBE.

Proposition 1 introduces the price self-sensitivity coefficient, the price cross-sensitivity coefficient, and the market share as the single scenario into the expression and indicates the impact on the CFBE. We can gain managerial insight from the proposition that the CFBE can be increased or decreased with the interaction effect and the market share relative to the situation without the interaction. Proposition (i) describes the cash flow bullwhip effect for "Retailer 1" in which the customer's demand only relies on the price self-sensitivity coefficient. From propositions (ii) and (iii), we can conclude that the cash flow bullwhip effect can be decreased when the price cross-sensitivity coefficient and the market share are introduced into the demand model. Proposition (ii) shows that only the price cross-sensitivity coefficient is introduced into the demand model and indicates that the CFBE can be decreased when the following condition holds:

$$
b_{12}^{2}\left(\delta_{2, p}\right)^{2} \Lambda_{2}\left(l_{2}-\Omega_{2}\right) k^{2}+l_{1}^{2}\left[b_{11}^{2}\left(\delta_{1, p}\right)^{2} \Lambda_{1}\left(k-\Theta_{1}\right)+b_{12}^{2}\right.
$$
$\left.\left(\delta_{2, p}\right)^{2} \Lambda_{2}\left(k-\Theta_{2}\right)\right]-l_{1} k\left\{b_{12} b_{22} \rho_{2} \Omega_{2} \Theta_{2}\left(\delta_{2, p}\right)^{2}\right\}>0$. From proposition (iii), we can observe that the market share can reduce the cash flow bullwhip effect under some conditions. Proposition (iv) indicates that when the price cross-sensitivity coefficient and the market share are introduced incrementally to "Scenario 1," the cash flow bullwhip effehct can be reduced very quickly. Proposition (v) indicates that the price crosssensitivity coefficient, the market share, and the covariance are introduced incrementally to "Scenario 1," the cash flow bullwhip effect can be increased under some conditions. Proposition (vi) suggests that using the interaction demand model and by introducing only the market share into the expression, the cash flow bullwhip effect has no change.

\section{Corollary 1. For the compounding cause of the CFBE:}

(i) The cash flow bullwhip effect can exist, it is smaller than that observed in the single $S C$ when satisfying the condition: $b_{12} b_{22} \rho_{2} \Omega_{2} \Theta_{2}\left(\delta_{2, p}\right)^{2}+b_{12}^{2}\left(\delta_{2, p}\right)^{2}$ $\Lambda_{2}\left(l_{2}-\Omega_{2}\right) k-b_{12}^{2}\left(\delta_{2, p}\right)^{2} \Lambda_{2}\left(k-\Theta_{2}\right) \leq b_{11}^{2}\left(\delta_{1, p}\right)^{2}$ $\Lambda_{1}\left(k-\Theta_{1}\right)$.

(ii) The cash flow bullwhip effect can exist, and it is larger than that observed in the single SC when satisfying the condition: $\left(b_{11}^{2}+b_{12}^{2}\right)\left(\delta_{1, p}\right)^{2} \Lambda_{1}\left(k-\Theta_{1}\right)>$ $b_{12} b_{22} \rho_{2} \Omega_{2} \Theta_{2}\left(\delta_{2, p}\right)^{2}+b_{12}^{2}\left(\delta_{2, p}\right)^{2} \Lambda_{2}\left(l_{2}-\Omega_{2}\right) k-b_{12}^{2}$ $\left(\delta_{2, p}\right)^{2} \Lambda_{2}\left(k-\Theta_{2}\right)$.

(iii) The combination of the price cross-sensitivity coefficient, the market share, and the covariance can reduce the CFBE when satisfying the condition:

$$
\left\{1+\frac{2\left(1+\Lambda_{1}\right)\left(\Lambda_{1}\right)\left\{b_{11} b_{12}\left(\rho_{1}^{k}+\rho_{2}^{k}\right) \delta_{12}^{2} \Delta\right\}}{\left(\delta_{1, d}\right)^{2}}\right\} \leq\left\{\left(\frac{S_{1}}{C_{1}}\right)^{2}+1\right\} \frac{\left\{\begin{array}{l}
2 b_{11} b_{12} \delta_{12}^{2} \Delta \Lambda_{1}\left(l_{1}-\Omega_{1}\right)+2 b_{12} b_{11} \delta_{12}^{2} \Delta \Lambda_{2}\left(l_{2}-\Omega_{2}\right) \\
+\left(l_{1} / k\right)^{2}\left[2 b_{11} b_{12}\left(\Lambda_{1}\left(k-\Theta_{1}\right)-\Lambda_{2}\left(k-\Theta_{2}\right)\right) \Delta \delta_{12}^{2}\right] \\
-2\left(l_{1} / k\right)\left\{b_{11} b_{22} \rho_{1} \Omega_{1} \Theta_{1} \delta_{12}^{2} \Delta+b_{12} b_{21} \rho_{2} \Omega_{2} \Theta_{2} \Delta \delta_{12}^{2}\right\}
\end{array}\right\}}{\left(\delta_{1, d}\right)^{2}} .
$$

Point (i) proves that the CFBE is smaller than that observed in a single SC because of the role of the price crosssensitivity coefficient. Point (ii) suggests that the CFBE is larger than that observed in a single SC because of the role of the market share. Point (iii) reveals that the combination of the price cross-sensitivity coefficient, the market share, and the covariance can reduce the CFBE.

\section{Numerical Simulation and Analysis}

In Section 5, we have analyzed the conditions under which the price self-sensitivity coefficient, the price cross-sensitivity coefficient, and the market share can reduce the CFBE in two-product and two-parallel SC systems compared with a single supply chain. Propositions (ii), (iii), (iv), and (v) have demonstrated the condition. Assuming that two supply chains have the same structure, we thoroughly explain the condition using the first supply chain. The relevant parameters can be used, $b_{11}=1, b_{21}=1, l_{1}=1, l_{2}=1, k=1$, $b_{12} \in\{1,2,3\}, \rho_{1} \in\{0,1\}, \rho_{2}=\in\{0,1\} .\left(\delta_{1, p}\right)^{2}=\left(\delta_{2, p}\right)^{2}=1$, $\delta_{12}^{2} \in\{0,0.1,0.2\}$. The simulation result is illustrated in Figures 2-4.

According to Figures 2-4, we can conclude the following:

(1) From Figure 2, if $b_{12}$ is nonnegative, the area of $\mathrm{CFBE}_{\text {retailer-1 }}^{\text {self }+ \text { cross }}>\mathrm{CFBE}_{\text {retailer-1 }}^{\text {self }}$ is increased when $b_{12}$ increases from 1 to 3 , and is decreased when $\delta_{12}^{2}$ increases from 0 to 0.2 .

(2) From Figure 3, if the value $\rho_{1} \in\{0,0.5\}$, the area of $\mathrm{CFBE}_{\text {retailer-1 }}^{\text {self }+ \text { market }}>\mathrm{CFBE}_{\text {retailer-1 }}^{\text {self }}$ is increased when $\psi$ increases from 0 to 0.5 . Otherwise, the area of $\mathrm{CFBE}_{\text {retailer-1 }}^{\text {self }+ \text { market }}>\mathrm{CFBE}_{\text {retailer-1 }}^{\text {self }}$ is decreased when $\psi$ increases from 0 to 0.5 .

(3) From Figure 4, when $b_{12}$ is nonnegative, if the value $\rho_{1} \in\{0,0.5\}$, the area of $\mathrm{CFBE}_{\text {retailer-1 }}^{\text {self }+ \text { cross+market }}$ 

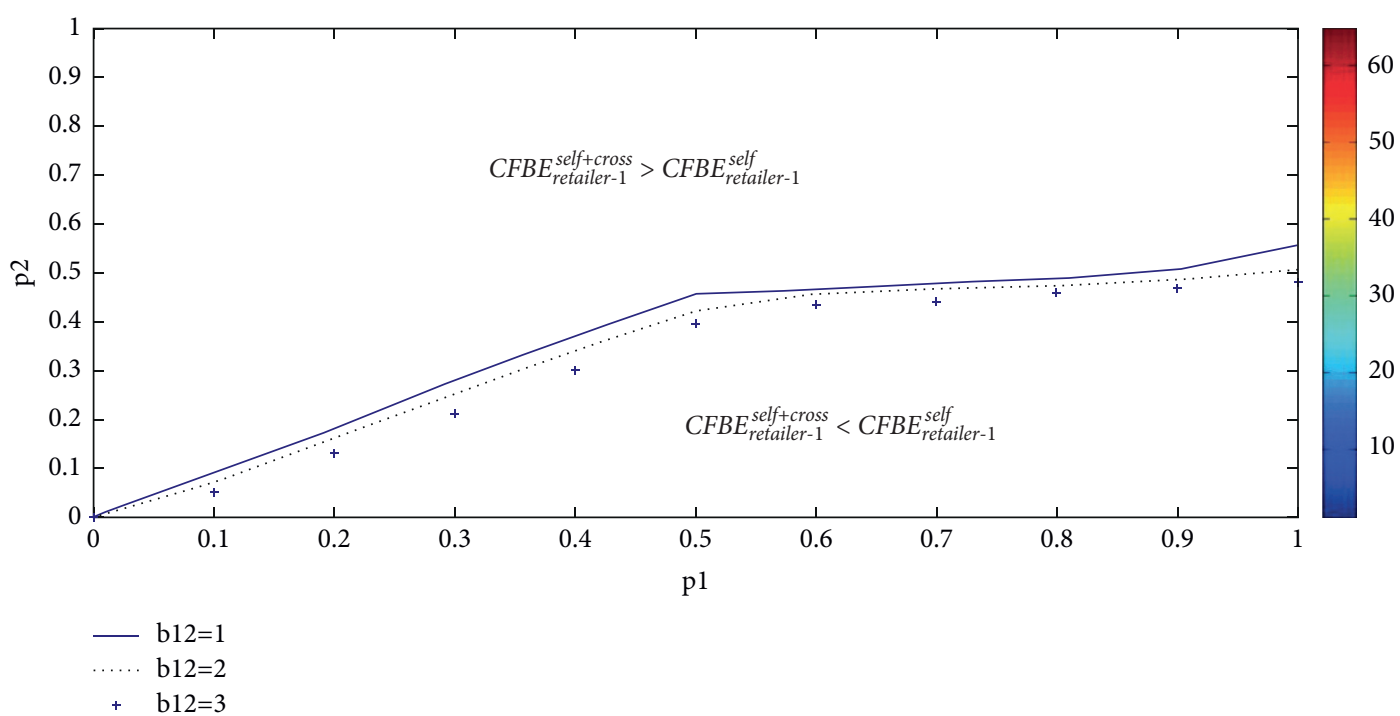

(a)

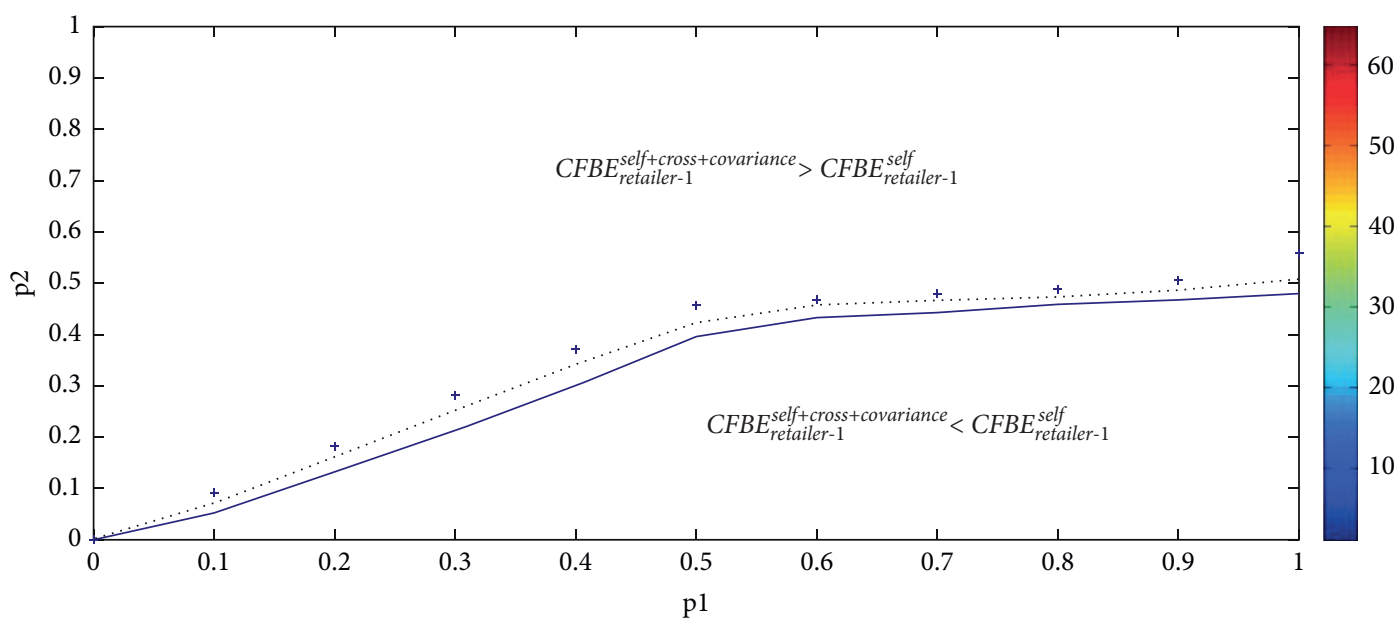

$$
\begin{aligned}
& -\delta 12^{2}=0 \\
& \ldots \ldots 12^{2}=0.1 \\
& +\quad \delta 12^{2}=0.2
\end{aligned}
$$

(b)

FIGURE 2: (a) The impact of $b_{12}$ on the $\mathrm{CFBE}_{\text {retailer-1 }}^{\text {self }+ \text { cross }}>\mathrm{CFBE}_{\text {retailer-1 }}^{\text {self }}$ and on the $\mathrm{CFBE}_{\text {retailer-1 }}^{\text {self }+ \text { cross }}<\mathrm{CFBE}_{\text {retailer-1 }}^{\text {self }}$ (b) The impact of $\delta_{12}^{2}$ on the $\mathrm{CFBE}_{\text {retailer-1 }}^{\text {self+covariance }}>\mathrm{CFBE}_{\text {retailer-1 }}^{\text {self }}$ and on the CFBE retailer-1 $^{\text {self }+ \text { crovariance }}<\mathrm{CFBE}_{\text {retailer- }-1}^{\text {self }}$.

$>\mathrm{CFBE}_{\text {retailer-1 }}^{\text {self }}$ is increased when $b_{12}$ increases from 1 to 3 . Otherwise, the area of $\mathrm{CFBE}_{\text {retailer-1 }}^{\text {self }+ \text { cross+market }}>\mathrm{CFBE}_{\text {retailer-1 }}^{\text {self }}$ is decreased when $b_{12}$ increases from 1 to 3 .

To sum up, the cash flow bullwhip effect increased with the increase in the price cross-sensitivity coefficient. If the value $\rho_{1}$ was lower than 0.5 , the cash flow bullwhip effect increased when the market share increased. Otherwise, the CFBE decreased when the market share increased. The cash flow bullwhip effect could be further amplified when the market share and the price cross-sensitivity coefficient were introduced into the demand model in the meantime. On the one hand, when the price cross-sensitivity coefficient was nonnegative, the covariance could increase the CFBE. On the other hand, the covariance could dampen the cash flow bullwhip effect.

From the section presented above, we found that the cash flow bullwhip effect increased in accordance with the increase in the price cross-sensitivity coefficient when the value $b_{12} \geq 1$. Thus, we discuss one question: whether the cash flow bullwhip effect is not observed when the value $b_{12} \in\{0,1\}$. To determine this answer, we established the 


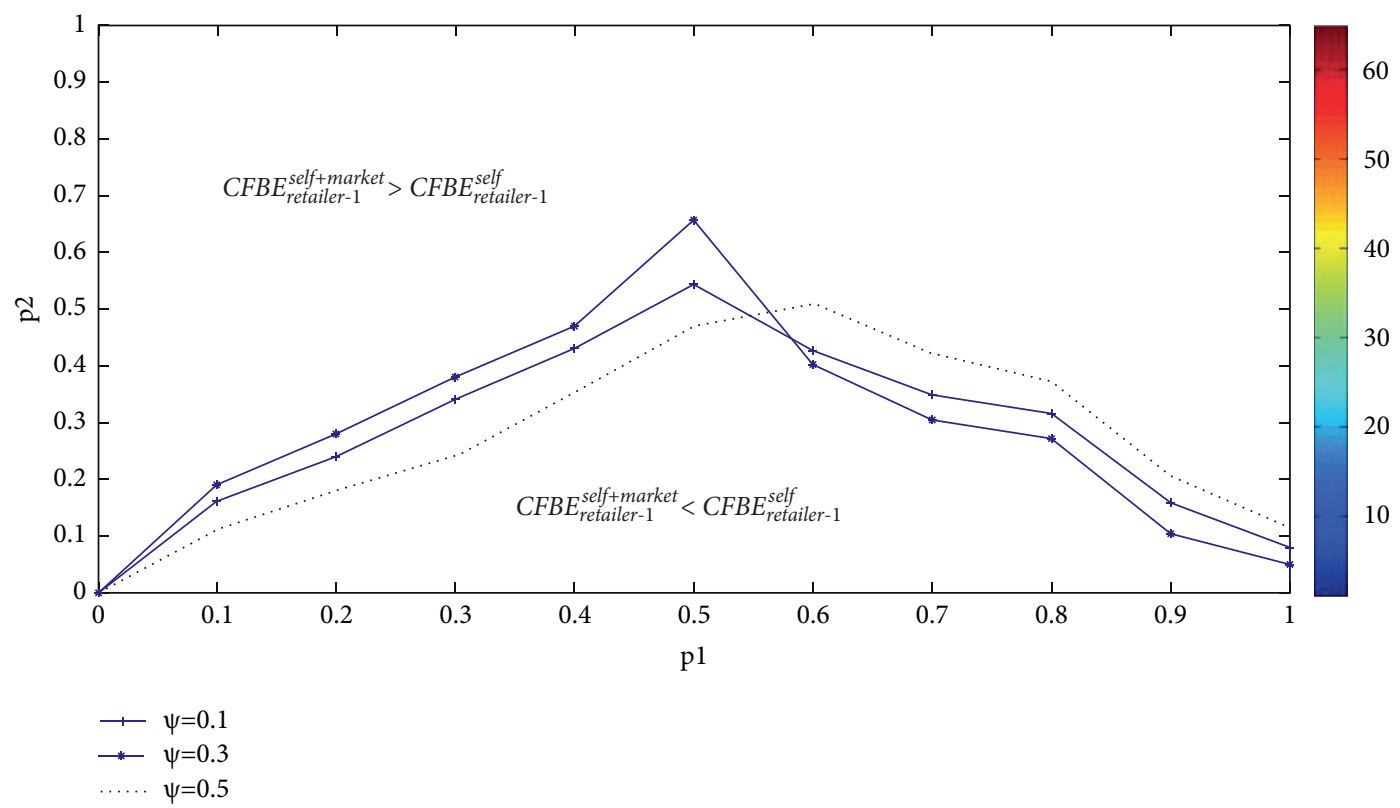

FIGURE 3: The impact of $b_{12}$ on the $\mathrm{CFBE}_{\text {retailer }-1}^{\text {self }+ \text { market }}>\mathrm{CFBE}_{\text {retailer-1 }}^{\text {self }}$ and on the $\mathrm{CFBE}_{\text {retailer }-1}^{\text {self }+ \text { market }}<\mathrm{CFBE}_{\text {retailer- } 1}^{\text {self }}$.

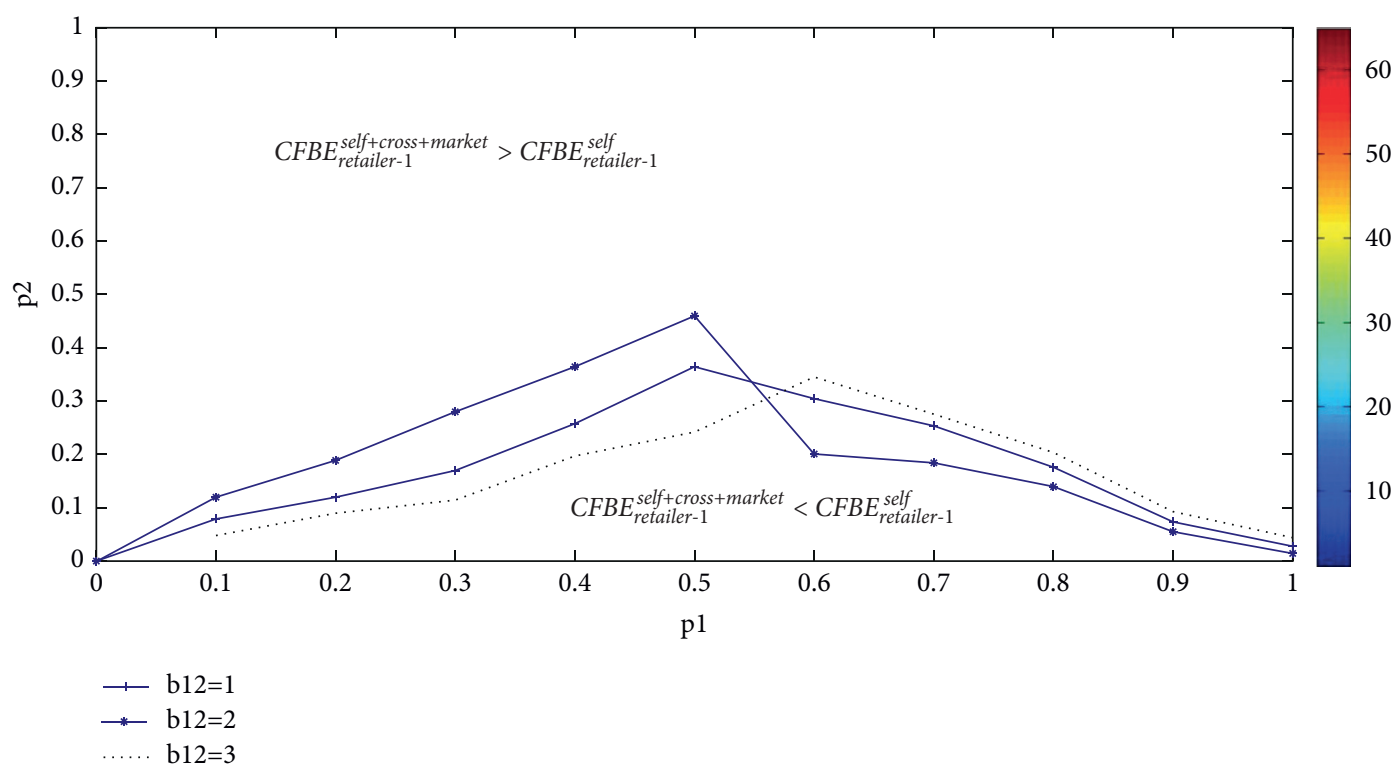

Figure 4: The impact of $b_{12}$ on the $\mathrm{CFBE}_{\text {retailer-1 }}^{\text {self }+ \text { cross+market }}>\mathrm{CFBE}_{\text {retailer-1 }}^{\text {self }}$ and on the $\mathrm{CFBE}_{\text {retailer-1 }}^{\text {self } 1 \text {-crosset }}<\mathrm{CFBE}_{\text {retailer-1 }}^{\text {self }}$.

TABLE 1: The values of CFBE when. $b_{12} \in\{0,1\}$

\begin{tabular}{ccccccccccccc}
\hline \multicolumn{10}{c}{$b_{12}$} \\
\hline & & 0 & 0.1 & 0.2 & 0.3 & 0.4 & 0.5 & 0.6 & 0.7 & 0.8 & 0.9 & 1 \\
& 0 & 1.12 & 1.15 & 1.18 & 1.21 & 1.28 & 1.31 & 1.45 & 1.54 & 1.64 & 1.84 & 1.94 \\
& 0.1 & 1.14 & 1.18 & 1.24 & 1.26 & 1.29 & 1.37 & 1.51 & 1.56 & 1.74 & 1.89 & 2.01 \\
$\rho_{1}=0.3, \rho_{2}=0.7$ & 0.2 & 1.21 & 1.28 & 1.29 & 1.31 & 1.34 & 1.39 & 1.64 & 1.74 & 1.85 & 1.91 & 2.12 \\
& 0.3 & 1.26 & 1.34 & 1.39 & 1.42 & 1.45 & 1.49 & 1.74 & 1.84 & 1.92 & 1.99 & 2.25 \\
& 0.4 & 1.28 & 1.39 & 1.42 & 1.58 & 1.61 & 1.74 & 1.89 & 1.95 & 2.01 & 2.21 & 2.34 \\
& 0.5 & 1.34 & 1.41 & 1.51 & 1.61 & 1.68 & 1.85 & 1.95 & 2.01 & 2.15 & 2.35 & 2.51 \\
& 0.6 & 1.45 & 1.49 & 1.67 & 1.74 & 1.78 & 1.91 & 2.14 & 2.23 & 2.25 & 2.41 & 2.67 \\
& 0.7 & 1.57 & 1.61 & 1.79 & 1.85 & 1.89 & 2.01 & 2.21 & 2.34 & 2.41 & 2.58 & 2.94 \\
& 0.8 & 1.67 & 1.72 & 1.84 & 1.91 & 1.98 & 2.24 & 2.34 & 2.45 & 2.58 & 2.64 & 3.12 \\
& 0.9 & 1.71 & 1.85 & 1.92 & 2.01 & 2.21 & 2.61 & 2.74 & 2.81 & 2.91 & 3.05 & 3.24 \\
& 1 & 1.84 & 1.92 & 2.04 & 2.12 & 2.34 & 2.74 & 2.81 & 2.91 & 3.01 & 3.25 & 3.47 \\
\hline
\end{tabular}


TABLE 1: Continued.

\begin{tabular}{|c|c|c|c|c|c|c|c|c|c|c|c|c|}
\hline \multicolumn{13}{|c|}{$b_{12}$} \\
\hline \multirow{11}{*}{$\rho_{1}=0.5, \rho_{2}=0.5$} & 0 & 1.17 & 1.21 & 1.38 & 1.41 & 1.57 & 1.61 & 1.74 & 1.84 & 2.04 & 2.34 & 3.57 \\
\hline & 0.1 & 1.24 & 1.28 & 1.41 & 1.46 & 1.59 & 1.74 & 1.81 & 1.96 & 2.27 & 2.57 & 3.64 \\
\hline & 0.2 & 1.31 & 1.35 & 1.48 & 1.51 & 1.64 & 1.81 & 1.95 & 2.14 & 2.34 & 2.61 & 3.87 \\
\hline & 0.3 & 1.36 & 1.42 & 1.54 & 1.62 & 1.75 & 1.92 & 2.04 & 2.28 & 2.57 & 2.74 & 3.94 \\
\hline & 0.4 & 1.38 & 1.49 & 1.61 & 1.78 & 1.81 & 2.04 & 2.18 & 2.34 & 2.64 & 2.84 & 3.99 \\
\hline & 0.5 & 1.41 & 1.58 & 1.74 & 1.81 & 1.98 & 2.14 & 2.28 & 2.48 & 2.87 & 2.91 & 4.18 \\
\hline & 0.6 & 1.52 & 1.67 & 1.82 & 1.84 & 2.04 & 2.28 & 2.31 & 2.67 & 2.94 & 3.04 & 4.29 \\
\hline & 0.7 & 1.67 & 1.72 & 1.94 & 2.01 & 2.15 & 2.38 & 2.47 & 2.81 & 3.04 & 3.27 & 4.47 \\
\hline & 0.8 & 1.74 & 1.84 & 2.04 & 2.15 & 2.48 & 2.47 & 2.58 & 2.97 & 3.28 & 3.48 & 4.52 \\
\hline & 0.9 & 1.79 & 1.91 & 2.17 & 2.28 & 2.58 & 2.64 & 2.89 & 3.47 & 3.47 & 3.57 & 4.61 \\
\hline & 1 & 1.86 & 2.04 & 2.25 & 2.37 & 2.64 & 2.87 & 2.94 & 3.64 & 3.97 & 4.08 & 4.89 \\
\hline \multirow{11}{*}{$\rho_{1}=0.7, \rho_{2}=0.3$} & 0 & 1.28 & 1.37 & 1.48 & 1.57 & 1.64 & 1.78 & 1.84 & 2.04 & 2.15 & 2.45 & 3.87 \\
\hline & 0.1 & 1.35 & 1.45 & 1.57 & 1.61 & 1.78 & 1.94 & 1.99 & 2.15 & 2.37 & 2.78 & 3.91 \\
\hline & 0.2 & 1.42 & 1.58 & 1.69 & 1.74 & 1.86 & 2.05 & 2.17 & 2.28 & 2.45 & 2.91 & 3.99 \\
\hline & 0.3 & 1.53 & 1.67 & 1.78 & 1.85 & 1.94 & 2.18 & 2.27 & 2.35 & 2.67 & 2.99 & 4.27 \\
\hline & 0.4 & 1.67 & 1.79 & 1.98 & 2.04 & 2.14 & 2.22 & 2.38 & 2.46 & 2.85 & 3.08 & 4.38 \\
\hline & 0.5 & 1.71 & 1.85 & 2.08 & 2.15 & 2.25 & 2.37 & 2.41 & 2.51 & 2.91 & 3.12 & 4.41 \\
\hline & 0.6 & 1.82 & 1.95 & 2.18 & 2.27 & 2.34 & 2.41 & 2.57 & 2.74 & 3.05 & 3.37 & 4.58 \\
\hline & 0.7 & 1.91 & 1.99 & 2.27 & 2.34 & 2.45 & 2.57 & 2.69 & 2.91 & 3.15 & 3.45 & 4.68 \\
\hline & 0.8 & 2.07 & 2.14 & 2.31 & 2.45 & 2.58 & 2.67 & 2.81 & 2.99 & 3.37 & 3.64 & 4.91 \\
\hline & 0.9 & 2.16 & 2.28 & 2.47 & 2.56 & 2.67 & 2.72 & 2.92 & 3.57 & 3.47 & 3.84 & 5.28 \\
\hline & 1 & 2.24 & 2.37 & 2.59 & 2.78 & 2.88 & 2.91 & 3.08 & 3.64 & 4.05 & 4.18 & 5.81 \\
\hline
\end{tabular}

parameters $b_{12} \in\{0,0.1,0.2,0.3,0.4,0.5,0.6,0.7,0.8,0.9,1\}$, and we considered three scenarios in respect to the combination of $\rho_{1}$ and $\rho_{2}: \rho_{1}=0.3, \rho_{2}=0.7 ; \rho_{1}=0.5, \rho_{, 2}=0.5$; $\rho_{1}=0.7, \rho_{, 2}=0.3$. The cash flow bullwhip effect is shown in Table 1.

Table 1 shows that the CFEB always exists in "Retailer 1" when $b_{12} \geq 0$. When it has the substitutable relationship between two products, the CFBE always existed because of the competition effect.

Some important managerial insights can be gained from the above numerical analysis. When two products were substitutable, the CFBE always existed and could not be reduced. Moreover, when two products shared the market, the CFBE could be reduced by increasing of market share when the value of the correlation coefficient was lower than 0.5. Otherwise, the CFBE increased as the market share increases.

\section{Conclusion}

In this study, we determined the impact of the price crosssensitivity coefficient and market share on the CFBE in twoproduct and two-parallel SC systems, which included one supplier and one retailer in each of the SC. Assume that two retailers ordered a product from two suppliers using the order-up-to strategy and two retailers predicted the customers demand using the MA technique. First, we built the equation of the CFBE based on the $\mathrm{BE}$ and in reference to inventory. Then, we obtain the influence of the price selfsensitivity coefficient and the market share on CFBE. We divided the above factors into the single scenario and the compounding scenario. Finally, we analyzed the condition that the competition effect and the market share could increase or decrease the CFBE. We can get the following conclusions:

(1) The CFBE increased in accordance with the increases in the price cross-sensitivity coefficient. If the value $\rho_{1}$ was lower than 0.5 , the CFBE increased when the market share increased. Otherwise, the CFBE decreased when the market share increased.

(2) The CFBE could be further amplified when the market share and the price cross-sensitivity coefficient were introduced into the demand model in the meantime. On the one hand, when the price crosssensitivity coefficient was nonnegative, the covariance could increase the CFBE. On the other hand, the covariance could dampen the CFBE.

(3) When two products were substitutable, the CFBE always existed and could not be reduced. Moreover, when two products shared the market, the cash flow bullwhip effect could be reduced by increasing of market share when the value of the correlation coefficient was lower than 0.5. Otherwise, the CFBE could be increased with the increase in the market share.

The managerial insights of our research is that (1) the manager should cooperate with their partner. If two products were substitutable or if two SC were competitors and place less emphasis on the competitive relationship and maintain a good cooperative relationship. (2) Enterprise should improve the forecasting accuracy of the customer's demand and improve the service quality. Thus, it could increase the market share and reduce the CFBE. (3) With the development of the artificial intelligence, big data, and block 
chain, the firm should improve the accuracy of the prediction by using the above technology.

The limitation about our paper is that (1) we only researched the CFBE in two-product and two-parallel SC systems. We also assume that the consumer's demand information is symmetry, we do not consider the asymmetric information between different point firms. (2) We assumed that the firm forecasted the lead time demand for both products using the MA forecasting technique. We do not consider the impact of other forecasting method on the CFBE. (3) We only consider the impact of MA forecasting technique on the CFBE in traditional SC. However, we do not discuss the influence of different techniques on the CFBE in mixed SC which consists of online selling channel and offline selling channel.
The future direction is that (1) we can discuss the CFBE in a SC network which includes multisupplier, multimanufacturer, multidistributor, and multiretailers. (2) We can examine how the firm predicts the customer's lead time demand using other forecasting methods. (3) Point out that many suppliers or retailers take advantage of electronic commerce developments to build their network of sales channels to sell products. We should discuss the impact of different sales channels on the CFBE.

\section{Appendix}

(1) The proof of equation (15) is as follows: after iterating computation into equation (2), we obtain

$$
\begin{aligned}
p_{1, t-1} & =\left(1+\rho_{1}+\cdots+\rho_{1}^{k-1}\right) \psi \mu_{1}+\rho_{1}^{k} p_{1, t-k-1}+\psi \rho_{1}^{k-1} \eta_{1, t-k}+\psi \rho_{1}^{k-2} \eta_{1, t-k+1}+\cdots+\psi \eta_{1, t-1} \\
& =\frac{1-\rho_{1}^{i+1}}{1-\rho_{1}} \psi \mu_{1}+\rho_{1}^{k} p_{1, t-k-1}+\sum_{j=0}^{k-1} \psi \rho_{1}^{k-1-j} \eta_{1, t-k+j} .
\end{aligned}
$$

Thus, we obtain

$$
\begin{aligned}
& \operatorname{Cov}\left(p_{1, t-1}, p_{1, t-k-1}\right)=\operatorname{Cov}\left(\frac{1-\rho_{1}^{i+1}}{1-\rho_{1}} \psi \mu_{1}+\rho_{1}^{k} p_{1, t-k-1}+\sum_{j=0}^{k-1} \psi \rho_{1}^{k-1-j} \eta_{1, t-k+j}, p_{1, t-k-1}\right)=\rho_{1}^{k} \operatorname{Var}\left(p_{1, t}\right) \\
& \operatorname{Cov}\left(p_{2, t-1}, p_{2, t-k-1}\right)=\operatorname{Cov}\left(\frac{1-\rho_{2}^{i+1}}{1-\rho_{2}}(1-\psi) \mu_{2}+\rho_{2}^{k} p_{2, t-k-1}+\sum_{j=0}^{k-1}(1-\psi) \rho_{2}^{k-1-j} \eta_{2, t-k+j}, p_{2, t-k-1}\right)=\rho_{2}^{k} \operatorname{Var}\left(p_{2, t}\right) \\
& \operatorname{Cov}\left(p_{1, t-1}, p_{2, t-k-1}\right)=\operatorname{Cov}\left(\frac{1-\rho_{1}^{i+1}}{1-\rho_{1}} \psi \mu_{1}+\rho_{1}^{k} p_{1, t-k-1}+\sum_{j=0}^{k-1} \psi \rho_{1}^{k-1-j} \eta_{1, t-k+j}, p_{2, t-k-1}\right)=\rho_{1}^{k} \frac{\psi(1-\psi)}{1-\rho_{1} \rho_{2}} \delta_{12}^{2} \\
& \operatorname{Cov}\left(p_{1, t-k-1}, p_{2, t-1}\right)=\operatorname{Cov}\left(\frac{1-\rho_{2}^{i+1}}{1-\rho_{2}} \psi \mu_{2}+\rho_{2}^{k} p_{2, t-k-1}+\sum_{j=0}^{k-1} \psi \rho_{2}^{k-1-j} \eta_{2, t-k+j}, p_{1, t-k-1}\right)=\rho_{2}^{k} \frac{\psi(1-\psi)}{1-\rho_{1} \rho_{2}} \delta_{12}^{2} \\
& \operatorname{Cov}\left(d_{1, t-1}, d_{1, t-k-1}\right)=b_{11}^{2} \rho_{1}^{k} \operatorname{Var}\left(p_{1, t}\right)+b_{12}^{2} \rho_{2}^{k} \operatorname{Var}\left(p_{2, t}\right)-b_{11} b_{12}\left(\rho_{1}^{k}+\rho_{2}^{k}\right) \frac{\psi(1-\psi)}{1-\rho_{1} \rho_{2}} \delta_{12}^{2} .
\end{aligned}
$$

(2) The proof of equation (16) is as follows: after iterating computation into equation (2), we obtain

$$
\begin{aligned}
p_{2, t-1} & =\left(1+\rho_{2}+\cdots+\rho_{2}^{k-1}\right)(1-\psi) \mu_{2}+\rho_{1}^{k} p_{1, t-k-1}+(1-\psi) \rho_{2}^{k-1} \eta_{2, t-k}+(1-\psi) \rho_{2}^{k-2} \eta_{2, t-k+1}+\cdots+(1-\psi) \eta_{2, t-1} \\
& =\frac{1-\rho_{2}^{i+1}}{1-\rho_{2}}(1-\psi) \mu_{2}+\rho_{2}^{k} p_{2, t-k-1}+\sum_{j=0}^{k-1}(1-\psi) \rho_{2}^{k-1-j} \eta_{2, t-k+j} .
\end{aligned}
$$

Thus, we obtain 


$$
\begin{aligned}
& \operatorname{Cov}\left(p_{1, t-1}, p_{1, t-k-1}\right)=\operatorname{Cov}\left(\frac{1-\rho_{1}^{i+1}}{1-\rho_{1}} \psi \mu_{1}+\rho_{1}^{k} p_{1, t-k-1}+\sum_{j=0}^{k-1} \psi \rho_{1}^{k-1-j} \eta_{1, t-k+j}, p_{1, t-k-1}\right)=\rho_{1}^{k} \operatorname{Var}\left(p_{1, t}\right), \\
& \operatorname{Cov}\left(p_{2, t-1}, p_{2, t-k-1}\right)=\operatorname{Cov}\left(\frac{1-\rho_{2}^{i+1}}{1-\rho_{2}}(1-\psi) \mu_{2}+\rho_{2}^{k} p_{2, t-k-1}+\sum_{j=0}^{k-1}(1-\psi) \rho_{2}^{k-1-j} \eta_{2, t-k+j}, p_{2, t-k-1}\right)=\rho_{2}^{k} \operatorname{Var}\left(p_{2, t}\right), \\
& \operatorname{Cov}\left(p_{2, t-1}, p_{1, t-k-1}\right)=\operatorname{Cov}\left(\frac{1-\rho_{2}^{i+1}}{1-\rho_{2}}(1-\psi) \mu_{2}+\rho_{2}^{k} p_{2, t-k-1}+\sum_{j=0}^{k-1}(1-\psi) \rho_{2}^{k-1-j} \eta_{2, t-k+j}, p_{1, t-k-1}\right)=\rho_{2}^{k} \frac{\psi(1-\psi) \delta_{12}^{2}}{1-\rho_{1} \rho_{2}} \\
& \operatorname{Cov}\left(p_{1, t-1}, p_{2, t-k-1}\right)=\operatorname{Cov}\left(\frac{1-\rho_{1}^{i+1}}{1-\rho_{1}} \psi \mu_{1}+\rho_{1}^{k} p_{1, t-k-1}+\sum_{j=0}^{k-1} \psi \rho_{1}^{k-1-j} \eta_{1, t-k+j}, p_{2, t-k-1}\right)=\rho_{1}^{k} \frac{\psi(1-\psi)}{1-\rho_{1} \rho_{2}} \delta_{12}^{2}, \\
& \operatorname{Cov}\left(d_{2, t-1}, d_{2, t-k-1}\right)=b_{21}^{2} \rho_{2}^{k} \operatorname{Var}\left(p_{2, t}\right)+b_{22}^{2} \rho_{1}^{k} \operatorname{Var}\left(p_{1, t}\right)-b_{21} b_{22}\left(\rho_{2}^{k}+\rho_{1}^{k}\right) \frac{\psi(1-\psi)}{1-\rho_{1} \rho_{2}} \delta_{12}^{2} .
\end{aligned}
$$

(3) The proof of equations (21) and (22) are as follows: In order to obtain the variance of $I_{1, t}$, we need to determine all of the variances and covariances as follows:

$$
\begin{aligned}
\operatorname{Var}\left(S_{1, t-l_{1}-1}\right)= & \operatorname{Var}\left(\frac{l_{1}}{k} \sum_{i=1}^{k} d_{1, t-l_{1}-1-i}+z_{1} \widehat{\xi}_{1, t-l_{1}-1}^{l_{1}}\right) \\
= & \left(\frac{l_{1}}{k}\right)^{2} \operatorname{Var}\left(\sum_{i=1}^{k} d_{1, t-l_{1}-1-i}\right)+z_{1}^{2} \operatorname{Var}\left(\hat{\xi}_{1, t-l_{1}-1}^{l_{1}}\right)+2 z_{1}\left(\frac{l_{1}}{k}\right) \operatorname{Cov}\left(\sum_{i=1}^{k} d_{1, t-l_{1}-1-i}, \hat{\xi}_{1, t-l_{1}-1}^{l_{1}}\right) \\
= & \left(\frac{l_{1}}{k}\right)^{2} \operatorname{Var}\left(\sum_{i=1}^{k} d_{1, t-l_{1}-1-i}\right)+z_{1}^{2} \operatorname{Var}\left(\hat{\xi}_{1, t-l_{1}-1}^{l_{1}}\right) \\
= & \left(\frac{l_{1}}{k}\right)^{2}\left\{k \operatorname{Var}\left(d_{1, t}\right)+2 b_{11}^{2}\left(\sum_{i=1}^{k-1} i \rho_{1}^{k-i}\right) \operatorname{Var}\left(p_{1, t}\right)+2 b_{12}^{2}\left(\sum_{i=1}^{k-1} i \rho_{2}^{k-i}\right) \operatorname{Var}\left(p_{2, t}\right)\right. \\
& \left.-2 b_{11} b_{12} \frac{\psi(1-\psi)}{1-\rho_{1} \rho_{2}} \delta_{12}^{2}\left(\left(\sum_{i=1}^{k-1} i \rho_{1}^{k-i}\right)+\left(\sum_{i=1}^{k-1} i \rho_{2}^{k-i}\right)\right)\right\} \\
& -2 b_{11} b_{12} \frac{\psi(1-\psi)}{1-\rho_{1} \rho_{2}} \delta_{12}^{2}\left(\left(\sum_{i=0}^{l_{1}+1}\left(l_{1}-i\right) \rho_{1}^{i+1}\right)+\left(\sum_{i=0}^{l_{1}-1}\left(l_{2}-i\right) \rho_{2}^{i+1}\right)\right) \\
& \left(l_{1}+1\right) \operatorname{Var}\left(d_{1, t}\right)+2 b_{11}^{2}\left(\sum_{i=0}^{l_{1}-1}\left(l_{1}-i\right) \rho_{1}^{i+1}\right) \operatorname{Var}\left(p_{1, t}\right)+2 b_{12}^{2}\left(\sum_{i=0}^{l_{2}-1}\left(l_{2}-i\right) \rho_{2}^{i+1}\right) \operatorname{Var}\left(p_{2, t}\right) \\
l_{1=1}+1 & \operatorname{Var}\left(d_{1, t-i}\right)+2 \operatorname{Cov}\left(d_{1, t-1}, d_{1, t-2}\right)+2 \operatorname{Cov}\left(d_{1, t-1}, d_{1, t-3}\right)+\cdots+2 \operatorname{Cov}\left(d_{1, t-l_{1}}, d_{1, t-l_{1}-1}\right)
\end{aligned}
$$


18

Discrete Dynamics in Nature and Society

$$
\begin{aligned}
\operatorname{Cov}\left(q_{1, t-l_{1}}, S_{1, t-l_{1}-1}\right)= & \operatorname{Cov}\left(S_{1, t-l_{1}}-S_{1, t-l_{1}-1}+d_{1, t-l_{1}-1}, S_{1, t-l_{1}-1}\right) \\
= & \operatorname{Cov}\left(\left(1+\frac{l_{1}}{k}\right) d_{1, t-l_{1}-1}-\left(\frac{l_{1}}{k}\right) d_{1, t-l_{1}-k-1}+z_{1}\left(\widehat{\xi}_{1, t-l_{1}}^{l_{1}}-\widehat{\xi}_{1, t-l_{1}-1}^{l_{1}}\right),\left(\frac{l_{1}}{k}\right) \sum_{i=1}^{k} d_{1, t-l_{1}-1-i}+z_{1} \hat{\xi}_{1, t-l_{1}-1}^{l_{1}}\right) \\
= & \left(1+\frac{l_{1}}{k}\right)\left(\frac{l_{1}}{k}\right) \operatorname{Cov}\left(d_{1, t-l_{1}-1}, \sum_{i=1}^{k} d_{1, t-l_{1}-1-i}\right)-\left(\frac{l_{1}}{k}\right)^{2} \operatorname{Cov}\left(d_{1, t-l_{1}-k-1}, \sum_{i=1}^{k} d_{1, t-l_{1}-1-i}\right) \\
= & \left(1+\frac{l_{1}}{k}\right)\left(\frac{l_{1}}{k}\right)\left\{b_{11}^{2}\left(\sum_{i=1}^{k} \rho_{1}^{i}\right) \operatorname{Var}\left(p_{1, t}\right)+b_{12}^{2}\left(\sum_{i=1}^{k} \rho_{2}^{i}\right) \operatorname{Var}\left(p_{2, t}\right)-b_{11} b_{12}\left(\left(\sum_{i=1}^{k} \rho_{1}^{i}\right)+\left(\sum_{i=1}^{k} \rho_{2}^{i}\right)\right) \frac{\psi(1-\psi)}{1-\rho_{1} \rho_{2}} \delta_{12}^{2}\right\} \\
& -\left(\frac{l_{1}}{k}\right)^{2}\left\{b_{11}^{2}\left(\sum_{i=1}^{k} \rho_{1}^{i}\right) \operatorname{Var}\left(p_{1, t}\right)+b_{12}^{2}\left(\sum_{i=1}^{k} \rho_{2}^{i}\right) \operatorname{Var}\left(p_{2, t}\right)-b_{11} b_{12}\left(\left(\sum_{i=1}^{k} \rho_{1}^{i}\right)+\left(\sum_{i=1}^{k} \rho_{2}^{i}\right)\right) \frac{\psi(1-\psi)}{1-\rho_{1} \rho_{2}} \delta_{12}^{2}\right\} \\
= & \left(1+\frac{l_{1}}{k}\right)\left(\frac{l_{1}}{k}\right)\left\{b_{11}^{2}\left(\frac{\rho_{1}\left(1-\rho_{1}^{k}\right)}{1-\rho_{1}}\right) \operatorname{Var}\left(p_{1, t}\right)+b_{12}^{2}\left(\frac{\rho_{2}\left(1-\rho_{2}^{k}\right)}{1-\rho_{2}}\right) \operatorname{Var}\left(p_{2, t}\right)-b_{11} b_{12}\left(\left(\frac{\rho_{1}\left(1-\rho_{1}^{k}\right)}{1-\rho_{1}}\right)+\left(\frac{\rho_{2}\left(1-\rho_{2}^{k}\right)}{1-\rho_{2}}\right)\right) \frac{\psi(1-\psi)}{1-\rho_{1} \rho_{2}} \delta_{12}^{2}\right\} \\
& -\left(\frac{l_{1}}{k}\right)^{2}\left\{b_{11}^{2}\left(\frac{\left(1-\rho_{1}^{k}\right)}{1-\rho_{1}}\right) \operatorname{Var}\left(p_{1, t}\right)+b_{12}^{2}\left(\frac{\left(1-\rho_{2}^{k}\right)}{1-\rho_{2}}\right) \operatorname{Var}\left(p_{2, t}\right)-b_{11} b_{12}\left(\left(\frac{\left(1-\rho_{1}^{k}\right)}{1-\rho_{1}}\right)+\left(\frac{\left(1-\rho_{2}^{k}\right)}{1-\rho_{2}}\right)\right) \frac{\psi(1-\psi)}{1-\rho_{1} \rho_{2}} \delta_{12}^{2}\right\},
\end{aligned}
$$

$$
\begin{aligned}
\operatorname{Cov}\left(q_{1, t-l_{1}}, d_{1, t}\right)= & \operatorname{Cov}\left(S_{1, t-l_{1}}-S_{1, t-l_{1}-1}+d_{1, t-l_{1}-1}, d_{1, t}\right) \\
= & \operatorname{Cov}\left(\left(1+\frac{l_{1}}{k}\right) d_{1, t-l_{1}-1}-\left(\frac{l_{1}}{k}\right) d_{1, t-l_{1}-k-1}+z_{1}\left(\hat{\xi}_{1, t-l_{1}}-\widehat{\xi}_{1, t-l_{1}-1}^{l_{1}}\right), d_{1, t}\right) \\
= & \left(1+\frac{l_{1}}{k}\right) \operatorname{Cov}\left(d_{1, t-l_{1}-1}, d_{1, t}\right)-\left(\frac{l_{1}}{k}\right) \operatorname{Cov}\left(d_{1, t-l_{1}-k-1}, d_{1, t}\right) \\
= & \left(1+\frac{l_{1}}{k}\right)\left(b_{11}^{2} \rho_{1}^{l_{1}+1} \operatorname{Var}\left(p_{1, t}\right)+b_{12}^{2} \rho_{2}^{l_{2}+1} \operatorname{Var}\left(p_{2, t}\right)-b_{11} b_{12}\left(\rho_{1}^{l_{1}+1}+\rho_{2}^{l_{2}+1}\right) \frac{\psi(1-\psi)}{1-\rho_{1} \rho_{2}} \delta_{12}^{2}\right) \\
& -\left(\frac{l_{1}}{k}\right)\left(b_{11}^{2} \rho_{1}^{l_{1}+k+1} \operatorname{Var}\left(p_{1, t}\right)+b_{12}^{2} \rho_{2}^{l_{2}+k+1} \operatorname{Var}\left(p_{2, t}\right)-b_{11} b_{12}\left(\rho_{1}^{l_{1}+k+1}+\rho_{2}^{l_{2}+k+1}\right) \frac{\psi(1-\psi)}{1-\rho_{1} \rho_{2}} \delta_{12}^{2}\right),
\end{aligned}
$$

$$
\begin{aligned}
\operatorname{Cov}\left(q_{1, t-l_{1}}, \sum_{i=1}^{l_{1}+1} d_{1, t-i}\right)= & \operatorname{Cov}\left(S_{1, t-l_{1}}-S_{1, t-l_{1}-1}+d_{1, t-l_{1}-1}, \sum_{i=1}^{l_{1}+1} d_{1, t-i}\right) \\
= & \operatorname{Cov}\left(\left(1+\frac{l_{1}}{k}\right) d_{1, t-l_{1}-1}-\left(\frac{l_{1}}{k}\right) d_{1, t-l_{1}-k-1}+z_{1}\left(\hat{\xi}_{1, t-l_{1}}^{l_{1}}-\hat{\xi}_{1, t-l_{1}-1}^{l_{1}}\right), \sum_{i=1}^{l_{1}+1} d_{1, t-i}\right) \\
= & \left(1+\frac{l_{1}}{k}\right) \operatorname{Cov}\left(d_{1, t-l_{1}-1}, \sum_{i=1}^{l_{1}+1} d_{1, t-i}\right)-\left(\frac{l_{1}}{k}\right) \operatorname{Cov}\left(d_{1, t-l_{1}-k-1}, \sum_{i=1}^{l_{1}+1} d_{1, t-i}\right) \\
= & \left(1+\frac{l_{1}}{k}\right)\left\{b_{11}^{2}\left(\sum_{i=0}^{l_{1}} \rho_{1}^{i}\right) \operatorname{Var}\left(p_{1, t}\right)+b_{12}^{2}\left(\sum_{i=0}^{l_{2}} \rho_{2}^{i}\right) \operatorname{Var}\left(p_{2, t}\right)-b_{11} b_{12}\left(\left(\sum_{i=0}^{l_{1}} \rho_{1}^{i}\right)+\left(\sum_{i=0}^{l_{2}} \rho_{2}^{i}\right)\right) \frac{\psi(1-\psi)}{1-\rho_{1} \rho_{2}} \delta_{12}^{2}\right\} \\
& -\left(\frac{l_{1}}{k}\right)\left\{b_{11}^{2}\left(\sum_{i=k}^{k+l_{1}} \rho_{1}^{i}\right) \operatorname{Var}\left(p_{1, t}\right)+b_{12}^{2}\left(\sum_{i=k}^{k+l_{2}} \rho_{2}^{i}\right) \operatorname{Var}\left(p_{2, t}\right)-b_{11} b_{12}\left(\left(\sum_{i=k}^{k+l_{1}} \rho_{1}^{i}\right)+\left(\sum_{i=k}^{k+l_{2}} \rho_{2}^{i}\right)\right) \frac{\psi(1-\psi)}{1-\rho_{1} \rho_{2}} \delta_{12}^{2}\right\} \\
= & \left(1+\frac{l_{1}}{k}\right)\left\{b_{11}^{2}\left(\frac{\left(1-\rho_{1}^{l_{1}+1}\right)}{1-\rho_{1}}\right) \operatorname{Var}\left(p_{1, t}\right)+b_{12}^{2}\left(\frac{\left(1-\rho_{2}^{l_{2}+1}\right)}{1-\rho_{2}}\right) \operatorname{Var}\left(p_{2, t}\right)-b_{11} b_{12}\left(\left(\frac{\left(1-\rho_{1}^{l_{1}+1}\right)}{1-\rho_{1}}\right)+\left(\frac{\left(1-\rho_{2}^{l_{2}+1}\right)}{1-\rho_{2}}\right)\right) \frac{\psi(1-\psi)}{1-\rho_{1} \rho_{2}} \delta_{12}^{2}\right\} \\
& -\left(\frac{l_{1}}{k}\right)\left\{b_{11}^{2}\left(\frac{\rho_{1}^{k}\left(1-\rho_{1}^{l_{1}}\right)}{1-\rho_{1}}\right) \operatorname{Var}\left(p_{1, t}\right)+b_{12}^{2}\left(\frac{\rho_{2}^{k}\left(1-\rho_{2}^{l_{2}}\right)}{1-\rho_{2}}\right) \operatorname{Var}\left(p_{2, t}\right)-b_{11} b_{12}\left(\left(\frac{\rho_{1}^{k}\left(1-\rho_{1}^{l_{1}}\right)}{1-\rho_{1}}\right)+\left(\frac{\rho_{2}^{k}\left(1-\rho_{2}^{l_{2}}\right)}{1-\rho_{2}}\right)\right) \frac{\psi(1-\psi)}{1-\rho_{1} \rho_{2}} \delta_{12}^{2}\right\},
\end{aligned}
$$

(A.9) 


$$
\begin{aligned}
\operatorname{Cov}\left(S_{1, t-l_{1}-1}, d_{1, t}\right) & =\operatorname{Cov}\left(\left(\frac{l_{1}}{k}\right) \sum_{i=1}^{k} d_{1, t-l_{1}-1-i}+z_{1} \vec{\xi}_{1, t-l_{1}-1}^{l_{1}}, d_{1, t}\right) \\
& =\left(\frac{l_{1}}{k}\right) \operatorname{Cov}\left(\sum_{i=1}^{k} d_{1, t-l_{1}-1-i}, d_{1, t}\right) \\
& =\left(\frac{l_{1}}{k}\right)\left\{b_{11}^{2}\left(\sum_{i=l_{1}+2}^{l_{1}+k+2} \rho_{1}^{i}\right) \operatorname{Var}\left(p_{1, t}\right)+b_{12}^{2}\left(\sum_{i=l_{2}+2}^{l_{2}+k+2} \rho_{2}^{i}\right) \operatorname{Var}\left(p_{2, t}\right)-b_{11} b_{12}\left(\left(\sum_{i=l_{1}+2}^{l_{1}+k+2} \rho_{1}^{i}\right)+\left(\sum_{i=l_{2}+2}^{l_{2}+k+2} \rho_{2}^{i}\right)\right) \frac{\psi(1-\psi)}{1-\rho_{1} \rho_{2}} \delta_{12}^{2}\right\} \\
& =\left(\frac{l_{1}}{k}\right)\left\{b_{11}^{2}\left(\sum_{i=l_{1}+2}^{i=l_{1}+k+2} \rho_{1}^{i}\right) \operatorname{Var}\left(p_{1, t}\right)+b_{12}^{2}\left(\sum_{i=l_{2}+2}^{i=l_{2}+k+2} \rho_{2}^{i}\right) \operatorname{Var}\left(p_{2, t}\right)-b_{11} b_{12}\left(\left(\sum_{i=l_{1}+2}^{i=l_{1}+k+2} \rho_{1}^{i}\right)+\left(\sum_{i=l_{2}+2}^{i=l_{2}+k+2} \rho_{2}^{i}\right)\right) \frac{\psi(1-\psi)}{1-\rho_{1} \rho_{2}} \delta_{12}^{2}\right\} \\
& =\left(\frac{l_{1}}{k}\right)\left\{b_{11}^{2}\left(\frac{\rho_{1}^{l_{1}+2}\left(1-\rho_{1}^{k}\right)}{1-\rho_{1}}\right) \operatorname{Var}\left(p_{1, t}\right)+b_{12}^{2}\left(\frac{\rho_{2}^{l_{2}+2}\left(1-\rho_{2}^{k}\right)}{1-\rho_{2}}\right) \operatorname{Var}\left(p_{2, t}\right)-b_{11} b_{12}\left(\left(\frac{\rho_{1}^{l_{1}+2}\left(1-\rho_{1}^{k}\right)}{1-\rho_{1}}\right)+\left(\frac{\rho_{2}^{l_{2}+2}\left(1-\rho_{2}^{k}\right)}{1-\rho_{2}}\right)\right) \frac{\psi(1-\psi)}{1-\rho_{1} \rho_{2}} \delta_{12}^{2}\right\},
\end{aligned}
$$

$$
\begin{aligned}
\operatorname{Cov}\left(d_{1, t}, \sum_{i=1}^{l_{1}+1} d_{1, t-i}\right) & =\operatorname{Cov}\left(d_{1, t}, d_{1, t-1}\right)+\operatorname{Cov}\left(d_{1, t}, d_{1, t-2}\right)+\cdots+\operatorname{Cov}\left(d_{1, t}, d_{1, t-l_{1}-1}\right) \\
& =\left\{b_{11}^{2}\left(\sum_{i=1}^{l_{1}+1} \rho_{1}^{i}\right) \operatorname{Var}\left(p_{1, t}\right)+b_{12}^{2}\left(\sum_{i=1}^{l_{2}+1} \rho_{2}^{i}\right) \operatorname{Var}\left(p_{2, t}\right)-b_{11} b_{12}\left(\left(\sum_{i=1}^{l_{1}+1} \rho_{1}^{i}\right)+\left(\sum_{i=1}^{l_{2}+1} \rho_{2}^{i}\right)\right) \frac{\psi(1-\psi)}{1-\rho_{1} \rho_{2}} \delta_{12}^{2}\right\} \\
& =\left\{b_{11}^{2}\left(\frac{\rho_{1}\left(1-\rho_{1}^{l_{1}+1}\right)}{1-\rho_{1}}\right) \operatorname{Var}\left(p_{1, t}\right)+b_{12}^{2}\left(\frac{\rho_{2}\left(1-\rho_{2}^{l_{2}+1}\right)}{1-\rho_{2}}\right) \operatorname{Var}\left(p_{2, t}\right)-b_{11} b_{12}\left(\left(\frac{\rho_{1}\left(1-\rho_{1}^{l_{1}+1}\right)}{1-\rho_{1}}\right)+\left(\frac{\rho_{2}\left(1-\rho_{2}^{l_{2}+1}\right)}{1-\rho_{2}}\right)\right) \frac{\psi(1-\psi)}{1-\rho_{1} \rho_{2}} \delta_{12}^{2}\right\}
\end{aligned}
$$

$$
\operatorname{Cov}\left(S_{1, t-l_{1}-1}, \sum_{i=1}^{l_{1}+1} d_{1, t-i}\right)=\operatorname{Cov}\left(\left(\frac{l_{1}}{k}\right) \sum_{i=1}^{k} d_{1, t-l_{1}-1-i}+z_{1} \widehat{\xi}_{1, t-l_{1}-1}^{l_{1}}, \sum_{i=1}^{l_{1}+1} d_{1, t-i}\right)
$$$$
=\left(\frac{l_{1}}{k}\right) \operatorname{Cov}\left(\sum_{i=1}^{k} d_{1, t-l_{1}-1-i}, \sum_{i=1}^{l_{1}+1} d_{1, t-i}\right)
$$$$
=\left\{\begin{array}{l}
b_{11}^{2}\left(\sum_{i=1}^{k} \sum_{j=1}^{l_{1}+1} \rho_{1}^{l_{1}+1+i-j}\right) \operatorname{Var}\left(p_{1, t}\right)+b_{12}^{2}\left(\sum_{i=1}^{k} \sum_{j=1}^{l_{2}+1} \rho_{1}^{l_{2}+1+i-j}\right) \operatorname{Var}\left(p_{2, t}\right) \\
-b_{11} b_{12}\left(\left(\sum_{i=1}^{k} \sum_{j=1}^{l_{1}+1} \rho_{1}^{l_{1}+1+i-j}\right)+\left(\sum_{i=1}^{k} \sum_{j=1}^{l_{2}+1} \rho_{1}^{l_{2}+1+i-j}\right)\right) \frac{\psi(1-\psi)}{1-\rho_{1} \rho_{2}} \delta_{12}^{2}
\end{array}\right\} .
$$

\section{Data Availability}

The data used to support the findings of this study are available from the corresponding author upon request.

\section{Conflicts of Interest}

The authors declare that they have no conflicts of interest.

\section{Authors' Contributions}

C. X. J. and J. Z. conceptualized the study and wrote the original study. Y. X. G. reviewed and edited the article.

\section{Acknowledgments}

This work was supported by the Philosophy and Social Science Research Project of University in Jiangsu Province (no. 2020SJA1029), the Project of China Scholarship Council (no. 201706980022), and the Outstanding Doctor Scientific Research Fund Project at Jiangsu Normal University. The authors thank International Science Editing (https://www. internationalscienceediting.com) for editing this manuscript.

\section{References}

[1] H. L. Lee, V. Padmanabhan, and S. Whang, "Information distortion in a supply chain: the bullwhip effect," Management Science, vol. 43, no. 4, pp. 546-558, 1997.

[2] F. Chen, Z. Drezner, J. K. Ryan, and D. Simchi-Levi, "Quantifying the bullwhip effect in a simple supply chain: the impact of forecasting, lead times, and information," Management Science, vol. 46, no. 3, pp. 436-443, 2000.

[3] F. Chen, J. K. Ryan, D. Simchi-Levi, and D. Smith-Levi, "The Impact of Exponential Smoothing Forecasts on the Bullwhip 
Effect," Naval Research Logistics, vol. 47, no. 4, pp. 269-286, 2000.

[4] H. T. Luong, "Measure of bullwhip effect in supply chains with autoregressive demand process," European Journal of Operational Research, vol. 180, no. 3, pp. 1086-1097, 2007.

[5] H. T. Luong and N. H. Phien, "Measure of bullwhip effect in supply chains: the case of high order autoregressive demand process," European Journal of Operational Research, vol. 183, no. 1, pp. 197-209, 2007.

[6] Q. Zhang, Q. L. Da, and H. C. Shen, "The evaluation about bullwhip effect and the information sharing under ARIMA (0, 1,1 ) demand model," Journal of Chinese Management Science, vol. 9, no. 6, pp. 1-6, 2001.

[7] X. F. Shi, L. Q. Zhang, and D. Y. Yang, "The impact of the information on the supply chain," Systems Engineering, vol. 20, no. 3, pp. 37-40, 2002.

[8] X. Zhang, "Delayed demand information and dampened bullwhip effect," Operations Research Letters, vol. 33, no. 3, pp. 289-294, 2005.

[9] H. W. P. Liu, "The simulation of the bullwhip effect in multilevel supply chain based on the ARMA $(1,1)$ demand model," Journal of System Simulation, vol. 34, no. 6, pp. 3253-3257, 2008.

[10] H. L. Lee, V. Padmanabhan, and S. Whang, "The bullwhip effect in supply chains," Sloan Management Review, vol. 38, no. 3, pp. 93-102, 1997b.

[11] J. W. Forrester, Industrial Dynamics, MIT Press and John Wiley \& Sons Inc, New York, NY, USA, 1961.

[12] H. L. Lee, C. S. Kut, and S. T. Christopher, "The value of information sharing in a two-level supply chain," Management science, vol. 46, no. 5, pp. 626-643, 2000.

[13] L. C. Alwan, J. J. Liu, and D. Q. Yao, "Stochastic characterization of upstream demand processes in a supply Chain," IEEE Transactions, vol. 35, no. 9, pp. 207-219, 2003.

[14] W. Hausman and J. Miyaoka, "How a base stock policy using stale forecasts provides supply chain benefits," Manufacturing and Service Operations Management, vol. 51, no. 10, pp. 3085-3116, 2004.

[15] H. Liu and P. Wang, "P. A. R. M. A $(1,1)$ based on the needs of multi-level supply chain bullwhip effect simulation," Journal of System Simulation, vol. 21, no. 6, pp. 3253-3257, 2008.

[16] G Gaalman and S. M. Disney, "State space investigation of the bullwhip problem with $\operatorname{ARMA}(1,1)$ demand processes," International Journal of Production Economics, vol. 104, no. 2, pp. 327-339, 2006.

[17] G. Gaalman and S. M. Disney, "On bullwhip in a family of order-up-to policies with $\operatorname{ARMA}(2,2)$ demand and arbitrary lead-times," International Journal of Production Economics, vol. 121, no. 2, pp. 454-463, 2009.

[18] H.-K. Kim and J. K. Ryan, "The cost impact of using simple forecasting techniques in a supply chain," Naval Research Logistics, vol. 50, no. 5, pp. 388-411, 2003.

[19] W. Holland and M. S. Sodhi, "Quantifying the effect of batch size and order errors on the bullwhip effect using simulation," International Journal of Logistics Research and Applications, vol. 7, no. 3, pp. 251-261, 2004.

[20] X. Zhang, "The impact of forecasting methods on the bullwhip effect," International Journal of Production Economics, vol. 88, no. 1, pp. 15-27, 2004.

[21] T. Hosoda and S. M. Disney, "On variance amplification in a three-echelon supply chain with minimum mean square error forecasting," Omega, vol. 34, no. 4, pp. 344-358, 2006.
[22] X. Zhao and J. Xie, "Forecasting errors and the value of information sharing in a supply chain," International Journal of Production Research, vol. 40, no. 2, pp. 311-335, 2002.

[23] Y. Ma, N. Wang, A. Che, Y. Huang, and J. Xu, "The bullwhip effect under different information-sharing settings: a perspective on price-sensitive demand that incorporates price dynamics," International Journal of Production Research, vol. 51, no. 10, pp. 3085-3116, 2013.

[24] Y. Ma, N. Wang, Z. He, J. Lu, and H. Liang, "Analysis of the bullwhip effect in two parallel supply chains with interacting price-sensitive demands," European Journal of Operational Research, vol. 243, no. 3, pp. 815-825, 2015.

[25] C. C. Holt, F. Modigliani, J. Muth, and H. Simon, Planning Production, Inventories and the Work Force, Prentice-Hall, Hoboken, NJ, USA, 1960.

[26] A. S. Blinder, "Retail inventory investment and business fluctuations," Booking Paper on Economics Activity, vol. 2, no. 43, pp. 443-505, 1981.

[27] A. S. Blinder, "Inventories and the structure of macro models," The American Economic Review, vol. 71, no. 21, pp. 11-16, 1981.

[28] M. Baganha and M. Cohen, "The stabilizing effect on inventory in supply chains," Operational Research, vol. 46, no. 13 , pp. $572-583,1998$.

[29] D. R. Towill, "Dynamic analysis of an inventory and order based production control system," International Journal of Production Research, vol. 15, no. 3, pp. 369-383, 2000.

[30] X. Y. Huang and Z. Lu, "Distribution-centre supply chain model and bullwhip effect $H_{\infty}$ control," Journal of Chinese Management Science, vol. 6, no. 3, pp. 86-93, 2003.

[31] S. M. Disney, D. R. Towill, and W. Van De Velde, "Variance amplification and the golden ratio in production and inventory control," International Journal of Production Economics, vol. 90, no. 3, pp. 295-309, 2004.

[32] R. G. Ingalls, B. L. Foote, and A. Krishnamoorthy, "Reducing the bullwhip effect in supply chains with control-based forecasting," International Journal of Simulation and Process Modelling, vol. 12, no. 1, pp. 90-110, 2005.

[33] Y. Feng and J. H. Ma, "The research on the bullwhip effect based on O-S control theory," Journal of Zhejiang University, vol. 11, no. 4, pp. 50-56, 2008.

[34] F. Costantino, G. DiGravio, A. Shaban, and M. Tronci, "SPC forecasting system to mitigate the Bullwhip effect and inventory variance in supply chains," Expert Systems with Applications, vol. 42, no. 5, pp. 1773-1787, 2015.

[35] M. Udenio, J. C. Fransoo, and R. D. Peels, "The bullwhip effect, and the credit crisis: empirical modelling of supply chain dynamics," International Journal of Production Economics, vol. 160, no. 2, pp. 34-46, 2015.

[36] F. Costantino, G. Di Gravio, A. Shaban, and M. Tronci, "The impact of information sharing and inventory control coordination on supply chain performances," Computers and Industrial Engineering, vol. 76, no. 4, pp. 292-306, 2014.

[37] X. Zhang and G. J. Burke, "Analysis of compound bullwhip effect causes," European Journal of Operational Research, vol. 210, no. 3, pp. 514-526, 2011.

[38] Y. Ma, N. Wang, A. Che, Y. Huang, and J. Xu, "The bullwhip effect on product orders and inventory: a perspective of demand forecasting techniques," International Journal of Production Research, vol. 51, no. 1, pp. 281-302, 2013.

[39] D. Gao, N. Wang, Z. He, and T. Jia, "The bullwhip effect in an online retail supply chain: a perspective of price-sensitive demand based on the price discount in e-commerce," IEEE 
Transactions on Engineering Management, vol. 40, no. 99, pp. 1-15, 2017.

[40] R. Tangsucheeva and V. Prabhu, "Modeling and analysis of cash-flow bullwhip in supply chain," International Journal of Production Economics, vol. 145, no. 1, pp. 431-447, 2013.

[41] M. Goodarzi, P. Makvandi, R. F. Saen, and M. D. Sagheb, "What are causes of cash flow bullwhip effect in centralized and decentralized supply chains?" Applied Mathematical Modelling, vol. 44, no. 2, pp. 640-654, 2017.

[42] X. G. Yuan, X. Q. Zhang, and D. L. Zhang, "Analysis of the impact of different forecasting techniques on the inventory bullwhip effect in two parallel supply chains with a competition effect," Journal of Engineering, vol. 2020, Article ID 2987218, 2020. 